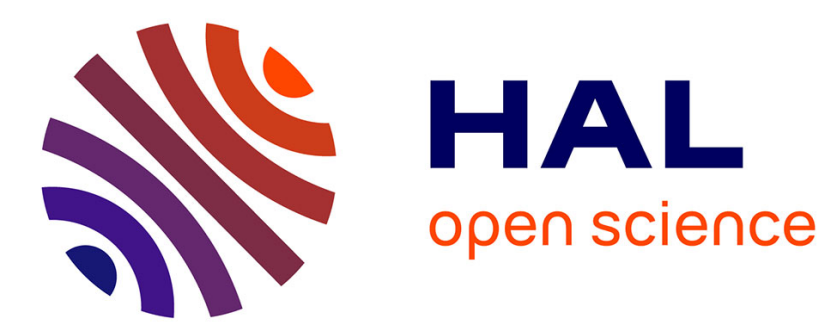

\title{
INTERFACE RESPONSE THEORY OF DISCRETE COMPOSITE SYSTEMS
}

\author{
L. Dobrzynski
}

\section{To cite this version:}

L. Dobrzynski. INTERFACE RESPONSE THEORY OF DISCRETE COMPOSITE SYSTEMS. Surface Science Reports, 1986, 6, pp.119-157. hal-03517064

\section{HAL Id: hal-03517064 \\ https://hal.archives-ouvertes.fr/hal-03517064}

Submitted on 7 Jan 2022

HAL is a multi-disciplinary open access archive for the deposit and dissemination of scientific research documents, whether they are published or not. The documents may come from teaching and research institutions in France or abroad, or from public or private research centers.
L'archive ouverte pluridisciplinaire HAL, est destinée au dépôt et à la diffusion de documents scientifiques de niveau recherche, publiés ou non, émanant des établissements d'enseignement et de recherche français ou étrangers, des laboratoires publics ou privés.

\section{(ㄷ)(1) $\$$}

Distributed under a Creative Commons Attribution - NonCommerciall 4.0 International 
Surface Science Reports 6 (1986) 119-157

North-Holland, Amsterdam

\section{INTERFACE RESPONSE THEORY OF DISCRETE COMPOSITE SYSTEMS}

\section{Leonard DOBRZYNSKI}

Centre National de la Recherche Scientifique, Laboratoire de Dynamique des Cristaux Moléculaires, Physique Fondamentale, Université des Sciences et Techniques de Lille, F-59655 Villeneuve D'Ascq Cedex, France

and

Consejo Superior de Investigaciones Cientificas, Instituto de Física del Estado Sólido, Serrano 123, Madrid 28006, Spain

Manuscript received in final form 13 February 1986

A general theory of "interface responses" in discrete composite $d$-dimensional systems for operators with two-body interactions is presented. It is shown that the "interface responses" of all the internal and external interfaces of any composite system are the linear superposition of the responses to a coupling operator of all individual interfaces and of the responses to a cleavage operator of the corresponding ideal free surfaces of the same but non-interacting subsystems. The response function and its elements between two space points of the system are given by a new simple general equation as a function of these "interface responses" and of the bulk response functions of each subsystem contained in the complete real system. The present paper establishes this new general two-body theory of interface responses for surfaces, interfaces, adsorbates, membranes, superlattices, defects of any kind and dimension, ... and for the first time, to the knowledge of the author, for any $d$-dimensional composite system. The presentation of the theory is followed here by a few general applications. 
To my wife

Marie-Françoise

and our children

Laetitia, Marie-Laure,

François and Coralie 


\section{Contents}

Preface

1. General introduction $\quad 124$

2. The theory $\quad 125$

$\begin{array}{ll}2.1 . & 125 \\ \end{array}$

2.2. Topology of a composite physical system 125

$\begin{array}{ll}\text { 2.2.1. Definitions } & 125\end{array}$

$\begin{array}{ll}\text { 2.2.2. Examples of systems with interfaces } & 126\end{array}$

2.3. Response functions in an infinite system $\quad 126$

2.3.1. Definitions $\quad 126$

$\begin{array}{ll}\text { 2.3.2. Examples } & 127\end{array}$

2.3.2.1. Examples of $d$-dimensional ensembles 127

$\begin{array}{ll}2.3 .2 .2 \text {. Examples of operators } & 127\end{array}$

2.4. One ideally truncated surface $\quad 127$

2.4.1. Definition of two independent ideally truncated systems $\quad 127$

$\begin{array}{ll}\text { 2.4.2. Surface response operators } & 127\end{array}$

$\begin{array}{lr}2.5 . & 129\end{array}$

$\begin{array}{ll}\text { 2.5.1. Definitions } & 129\end{array}$

2.5.2. Two-surfaces response operators 130

2.5.3. $J$-surfaces response operators $\quad 130$

2.6. Interfaces between two different subsystems

2.6.1. Definitions 131

2.6.2. Interface response operators $\quad 132$

2.6.3. $J$ interfaces between two different subsystems $\quad 133$

2.7. One adsorbed system on another one 133

$\begin{array}{ll}\text { 2.7.1. Definitions } & 133\end{array}$

$\begin{array}{ll}\text { 2.7.2. Response operators } & 134\end{array}$

2.7.3. Disconnected adsorptions of one subsystem on another one 135

2.7.3.1. Adsorption on $K$ disconnected voids 135

$\begin{array}{ll}\text { 2.7.3.2. Adsorption of } K \text { clusters } & 135\end{array}$

2.8. One membrane between two different systems $\quad 136$

2.8.1. Definitions 136

$\begin{array}{ll}\text { 2.8.2. Membrane response operators } & 137\end{array}$

2.8.3. $K$ disconnected membranes $\quad 138$

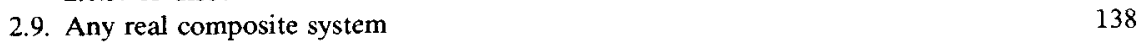

2.10.Different methods for including defects in a real system $\quad 139$

2.10.1. The bulk-surface method for defects $\quad 139$

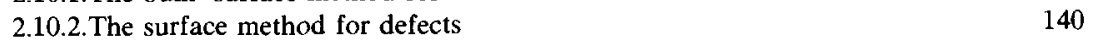

2.10.3. The perturbed bulk method for defects in composite systems $\quad 140$

$\begin{array}{ll}\text { 2.11. The alternative general equation } & 141\end{array}$

3. A few general applications $r$

3.1. Introduction $\quad 142$

$\begin{array}{ll}\text { 3.2. The elements of the response function } & 143\end{array}$

3.2.1. General relations 143

3.2.2. Summary and other relations between $\boldsymbol{g}$ and $\boldsymbol{G} \quad 144$

$\begin{array}{ll}\text { 3.3. Interface states } & 144\end{array}$

$\begin{array}{ll}\text { 3.4. Density of states } & 145\end{array}$

3.4.1. Total densities of states versus response functions $\quad 145$

$\begin{array}{ll}\text { 3.4.2. Local densities of states } & 146\end{array}$ 
3.4.3. Variation of the total density of states $\quad 146$

3.4.3.1. General result 146

3.4.3.2. Particular results $\quad 149$

3.4.3.2.1. In the complementary to $\mathrm{D}_{i}^{(d)}$ space 149

3.4.3.2.2. The casc of spccular symmetry through $\mathrm{M}_{i}^{(d)} \quad 150$

3.4.3.2.3. When one uses only surface response functions in $\boldsymbol{G} \quad 150$

3.4.3.3. Conservation of the number of states 150

3.5. Variations of additive functions $\quad 151$

3.6. Interface reflection and transmission $\quad 151$

3.7. Response to an external stimulus 153

3.8. Symmetry and super-periodicity 153

3.8.1. Symmetries $\quad 153$

$\begin{array}{ll}\text { 3.8.2. Interface super-periodicities } & 154\end{array}$

$\begin{array}{lr}\text { 4. Prospectives } & 156\end{array}$

Short literature survey on crystal interface response functions $\quad 156$

$\begin{array}{lr}\text { Acknowledgements } & 156\end{array}$

$\begin{array}{lr}\text { References } & 157\end{array}$ 


\section{Preface}

The present issue of Surface Science Reports is different in character compared with previous issues of the Journal. The manuscript is written in the style of a first chapter of a monograph on "Interface Responses". It presents a new general and simple theory of "Interface Responses" in discrete composite $d$-dimensional systems for operators with two-body interactions.

This self-contained text can be read even by undergraduate students, without need for interruption to consult references. However a short literature survey on crystal interface response functions is given at the end. 


\section{General introduction}

Consider any composite system and an operator $\boldsymbol{h}$, both defined in a $d$-dimensional discrete space. The inverse $\boldsymbol{g}$ of this operator is usually called response or Green function associated to $\boldsymbol{h}$.

When the operator $\boldsymbol{h}$ describes two-body interactions within the system, then the response function $\boldsymbol{g}$ is a function of two space positions $\boldsymbol{x}$ and $\boldsymbol{x}^{\prime}$. Its complete knowledge is equivalent to the complete knowledge of the operator $\boldsymbol{h}$. However a complete knowledge of $\boldsymbol{h}$ and of all its properties is not easy in a composite system. On the other hand, it is much easier to calculate and to understand all the properties of the corresponding bulk and ideal free surface operators and response functions for each of the homogeneous subsystems of the composite system.

The bulk response function of one subsystem has inside the space of definition of this subsystem the elements of the response function of the corresponding infinite homogeneous subsystem.

The ideally cleaved free surface response function of one subsystem can be obtained directly for systems with a small number of particles or from the corresponding bulk response function once one defines the ideal truncation of the corresponding infinite system.

A reference response function $\boldsymbol{G}$ can be defined for any composite system as a block diagonal operator. Each of the independent blocks of this operator is either the bulk or the ideal surface response function of the corresponding subsystem inside its space of definition.

The present theory relates the response function $\mathbf{g}$ of any composite system to its reference block diagonal response function $\boldsymbol{G}$, through two equivalent universal equations

$\boldsymbol{g}(\boldsymbol{I}+\boldsymbol{A})=\boldsymbol{G}$,

or

$\left(\boldsymbol{I}+\boldsymbol{A}^{\prime}\right) \boldsymbol{g}=\mathbf{G}$.

The "interface response" operators $\boldsymbol{A}$ and $\boldsymbol{A}^{\prime}$ are completely defined from the knowledge of the reference response function $\boldsymbol{G}$ and from those of the two-body interactions of the operator $\boldsymbol{h}$ at all the interfaces, $\boldsymbol{I}$ is the unity operator.

An easy way to construct these "interface response" operators consists in constructing the composite system out of the corresponding non-interacting subsystems. First one may define for each subsystem a cleavage operator which cuts in an infinite subsystem, the ideally cleaved free surface subsystem one needs. Then one binds these independent subsystems by coupling operators. These cleavage and coupling operators are easily written down once one knows the interaction at all the interfaces. 
The knowledge of the reference response function $\boldsymbol{G}$ and of the cleavage operators enables the "ideal surface response operators" to be written down. In a similar manner are defined the "surface-interface coupling response operators". And the "interface response" operators $\boldsymbol{A}$ and $\boldsymbol{A}^{\prime}$ are just the linear superposition of these ideal surface and surface-interface coupling response operators.

Two slightly different and simpler methods for including localized defects in any composite system are also proposed within the frame of the same theory.

It is well known that the bulk and surface response functions associated with operators like Hamiltonians, dynamical matrices,..., contain all the physical information associated with these operators and these systems; for example: the spectrum of excitations in condensed matters, the response to applied stimulus, .... In the same manner, the response functions $\boldsymbol{g}$ associated to an operator $\boldsymbol{h}$ of a composite system enables one to work out all physical properties of this system associated to this operator.

The general theory will be established in the next section. Then a few general applications will be given in section 3 .

\section{The theory}

\subsection{Introduction}

The two general equivalent equations of "interface response" theory will be demonstrated in this section 2 for an operator of any composite system contained in a discrete $d$-dimensional space.

Section 2.2 is devoted to a few simple considerations about the topology of composite systems and section 2.3 to the definition of response functions in infinite systems. These basic definitions enables then the "interface response operators" and response functions to be addressed for: one ideally truncated surface (section 2.4), several ideally truncated surfaces (section 2.5), interfaces between two different subsystems (section 2.6), one adsorbed system on another one (section 2.7), one membrane between two different systems (section 2.8), any composite system (section 2.9), defects of any kind and dimension in a composite system (section 2.10).

Finally, in section (2.11) the alternative equivalent general equation of this theory will be established.

\subsection{Topology of a composite physical system}

\subsubsection{Definitions}

Consider any composite system. It is contained in a finite domain $\mathrm{D}^{(d)}$ and can be considered as being made up of $N$ different subsystems. Each of these 
subsystems is contained in a subdomain $\mathrm{D}_{i}^{(d)}, 1 \leqq i \leqq N$. In what follows $\mathrm{D}_{i}^{(d)}$ is a discrete-in-space domain.

Define at the periphery of each domain $\mathrm{D}_{i}^{(d)}$ an interface domain $\mathrm{M}_{i}^{(d)}$. The ensemble of all $\mathbf{M}_{i}^{(d)}$ will be called the interface domain $\mathbf{M}^{(d)}$. When the subdomain $\mathrm{D}_{i}^{(d)}$ is made up of disconnected pieces or is in contact with several other subdomains or pieces of subdomains, then its interface domain $\mathbf{M}_{i}^{(d)}$ is composed of $J$ interfaces $\mathbf{M}_{i j}^{(d)}, 1 \leqq j \leqq J$.

The position of: all interfaces $\mathbf{M}_{i j}^{(d)}(1 \leqq i \leqq N, 1 \leqq j \leqq J)$ within the interface $\mathbf{M}_{i}^{(d)}$, of all interfaces $\mathbf{M}_{i}^{(d)}$ within the total interface domain $\mathbf{M}^{(d)}$ and of $\mathbf{M}^{(d)}$ and all $\mathrm{D}_{i}^{(d)}$ within the domain of existence $\mathrm{D}^{(d)}$ of the system under consideration defines the region of existence in $\mathrm{D}_{\infty}^{(d)}$ of the real system under consideration.

Note that an interface is a boundary domain and that it is defined in a space having the same dimensions as the domain this interface bounds.

\subsubsection{Examples of systems with interfaces}

In classical 3D geometry a volume is bounded by a 2D surface. This notion of a surface is generalized here and called an interface of zero thickness. The widely used notion of a $2 \mathrm{D}$ surface bounding a $3 \mathrm{D}$ volume is purely fictitious in physics. Any real physical system considered in real 3D space is bound by a 3D interface, which can be viewed as a surface of finite thickness. Note that this definition of an interface contains the classical definition of a $2 \mathrm{D}$ surface in a $3 \mathrm{D}$ geometry.

In what follows, the word surface will be reserved to ideally cleaved free surfaces. When the constituent properties of the system will be changed near the surface from their bulk values in a domain $\mathbf{M}_{i}^{(d)}$, the name interface will be used for this perturbed surface.

\subsection{Response functions in an infinite system}

\subsubsection{Definitions}

Consider any operator $\boldsymbol{H}_{0}$ defined in the infinite $d$-dimensional space $\mathrm{D}_{\infty}^{(d)}$. This means that the $d$ component discrete position $\boldsymbol{x}$ in this space is defined in the infinite $d$-dimensional volume of $\mathrm{D}_{\infty}^{(d)}$.

Define the response function $\boldsymbol{G}_{0}$ associated to $\boldsymbol{H}_{0}$ by

$\boldsymbol{H}_{0} \cdot \boldsymbol{G}_{0}=\boldsymbol{I}, \quad$ in $\mathrm{D}_{\infty}^{(d)}$,

where $I$ is the unity operator.

In what follows we will consider the same operators $\boldsymbol{H}_{0}$ and $\boldsymbol{G}_{0}$ for $N$ different systems. $\boldsymbol{H}_{0}$ and $\boldsymbol{G}_{0}$ will therefore be labelled by an index $1 \leqq i \leqq N$, and will be written as $\boldsymbol{H}_{0}(i, i)$ and $\boldsymbol{G}_{0}(i, i)$, both defined in $\mathrm{D}_{\infty}^{(d)}$. 


\subsubsection{Examples}

2.3.2.1. Examples of d-dimensional ensembles. The 1D, 2D and 3D real spaces are common. The $4 \mathrm{D}$ space when one adds the time to the three real space coordinates is also classical in relativity theory. The $d>4$ spaces used in phase transition theories and in statistical mechanics are more sophisticated.

Let us insist here that in the theory which follows the position vector $\boldsymbol{x}$ in any $d$-dimensional space is a discrete variable.

2.3.2.2. Examples of operators. Two common examples of operators in time invariant response theories are: Hamiltonians for electrons, dynamical matrices for phonons, defined in 1D, 2D or 3D real spaces.

In relativity theory, operators can be defined in the 4D space, which adds the time $t$ to the three real space components of $\boldsymbol{x}$.

\subsection{One ideally truncated surface}

\subsubsection{Definition of two independent ideally truncated systems}

Start from an infinite system contained in $\mathrm{D}_{\infty}^{(d)}$. Assume that the range of interactions between the particles and the fields contained in $\mathrm{D}_{\infty}^{(d)}$ and entering in the operator $\boldsymbol{H}_{0}$ is limited in space. Then, by cleavage create two independent subensembles $\mathrm{D}_{1}^{(d)}$ and $\mathrm{D}_{3}^{(d)}$. In order that these two systems be without mutual interactions, one has to cut all interactions between their two boundary domains or interfaces $\mathrm{M}_{1}^{(d)}$ and $\mathrm{M}_{3}^{(d)}$, whose extension in space depends on the range of mutual interactions of the particles and the fields.

One of these two independent subensembles may be finite and the other semi-infinite in space; or both may be semi-infinite. Fig. 1 illustrates these considerations in $2 \mathrm{D}$ real space.

\subsubsection{Surface response operators}

The cleavage described in section 2.4 .1 is represented by an operator $\mathbf{V}_{0}$. Then $\boldsymbol{H}_{0}$ becomes

$\boldsymbol{h}_{0}=\boldsymbol{H}_{0}+\boldsymbol{V}_{0}$, in $\mathrm{D}_{\infty}^{(d)}$,

although

$\boldsymbol{V}_{0}\left(\boldsymbol{x}, \boldsymbol{x}^{\prime}\right)=0$, for $\left\{\boldsymbol{x}, \boldsymbol{x}^{\prime}\right\} \notin\left\{\mathrm{M}_{1}^{(d)}, \mathrm{M}_{3}^{(d)}\right\}$,

where we use the well know mathematical symbols: \{\} for ensemble, $\in$ for belonging to and $\notin$ for not belonging to.

Define the corresponding resolvant opcrator $\boldsymbol{g}_{0}$ by

$\boldsymbol{h}_{0} \cdot \boldsymbol{g}_{0}=\boldsymbol{l}$, in $\mathrm{D}_{\infty}^{(d)}$,

then define

$A_{0}=V_{0} G_{0}$, in $D_{\infty}^{(d)}$. 


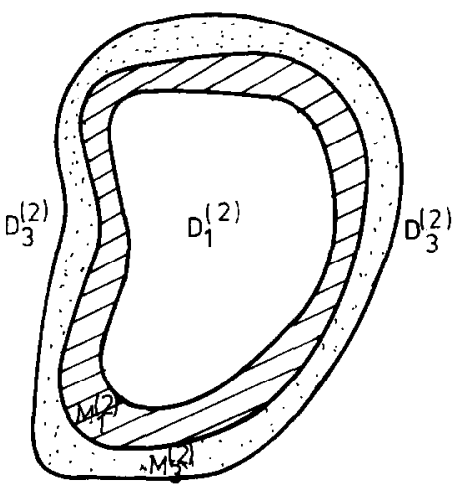

a

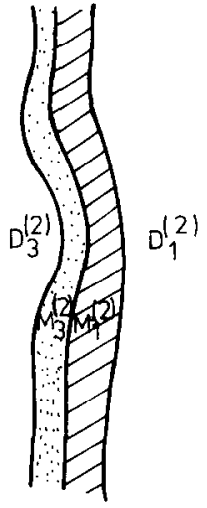

b

Fig. 1. Domains $\mathrm{D}_{1}^{(2)}$ and $\mathrm{D}_{3}^{(2)}$ bounded respectively by their "interfaces" $\mathbf{M}_{1}^{(2)}$ and $\mathbf{M}_{3}^{(2)}$; $M_{1}^{(2)} \in D_{1}^{(2)}$ and $M_{3}^{(2)} \in D_{3}^{(2)}$. The interface domain $M^{(2)} \equiv\left\{M_{1}^{(2)}, M_{3}^{(2)}\right\}$. (a) The case of one finite domain $D_{1}^{(2)}$ in contact with a semi-infinite one $D_{3}^{(2)}$ and (b) the two domains in contact are semi-infinite.

These definitions, together with those of eq. (2.1) lead directly to $\boldsymbol{G}_{0} \cdot\left(\boldsymbol{I}+\boldsymbol{A}_{0}\right)=\boldsymbol{G}_{0}, \quad$ in $\mathrm{D}_{\infty}^{(d)}$.

After cleavage

$\boldsymbol{h}_{0}\left(\boldsymbol{x}, \boldsymbol{x}^{\prime}\right)=0$ for $\left\{\boldsymbol{x}, \boldsymbol{x}^{\prime}\right\} \notin \mathrm{D}_{i}^{(d)}, i=1$ or 3 .

The two independent systems cannot respond to another, and

$\boldsymbol{g}_{0}\left(\boldsymbol{x}, \boldsymbol{x}^{\prime}\right)=0$ for $\left\{\boldsymbol{x}, \boldsymbol{x}^{\prime}\right\} \notin \mathrm{D}_{i}^{(d)}, i=1$ or 3.

So, eq. (2.5) can be written as

$\boldsymbol{g}_{\mathrm{s}} \cdot\left(\boldsymbol{I}+\boldsymbol{A}_{\mathrm{s}}\right)=\boldsymbol{G}_{\mathrm{s}}, \quad$ in $\mathrm{D}_{i}^{(d)}, \mathrm{i}=1$ or 3 .

In this equation enter only elements of $\boldsymbol{g}_{0}, \boldsymbol{A}_{0}$ and $\boldsymbol{G}_{0}$ within the same domain $\mathrm{D}_{i}^{(d)}$, although the calculation of the elements of $\boldsymbol{A}_{\mathrm{s}}$ defined in $\mathrm{D}_{i}^{(d)}$ has to be done from eq. (2.4) within $\mathrm{D}_{\infty}^{(d)}$.

In what follows $\boldsymbol{A}_{s}$ is called the ideal surface response operator and is such that

$\boldsymbol{A}_{\mathrm{s}}\left(\boldsymbol{x}, \boldsymbol{x}^{\prime}\right) \equiv 0 \quad$ for $\quad \boldsymbol{x} \notin \mathrm{M}_{i}^{(d)}$,

because of eqs. (2.2b) and (2.4).

In what follows this surface response operator $\boldsymbol{A}_{\mathrm{s}}$ defined in $\mathrm{D}_{i}^{(d)}$ will enter the calculation of the interface response operators of all the composite systems. Call it therefore

$\boldsymbol{A}_{s}(i, i), \quad$ in $\mathrm{D}_{i}^{(d)}$. 
And in the case where a system has $J$ disconnected free surfaces, the elements of $\boldsymbol{A}_{\mathrm{s}}(i, i)$ between for example the interfaces $\mathbf{M}_{i j}^{(d)}$ and $\mathbf{M}_{i j^{\prime}}^{(d)}$ are needed and are written

$\mathbf{A}_{\mathrm{s}}\left(i j, i j^{\prime}\right)$.

The same type of notation will be used for the other operators which appear in this theory.

\subsection{Ideally truncated surfaces}

\subsubsection{Definitions}

Start from an infinite system contained in $\mathrm{D}_{\infty}^{(d)}$. As described in section 2.4, create now in $\mathrm{D}_{2}^{(d)}$ one infinite system bounded by two disconnected "interfaces" $M_{21}^{(d)}$ and $M_{22}^{(d)}$ to two empty domains $D_{1}^{(d)}$ and $D_{3}^{(d)}$. Fig. 2a illustrates this in two dimensions, in the general case of two interfaces of finite volume. The case of a slab having two parallel interfaces is the other possibility depicted by fig. $2 b$.

Remember that here the interface domain is

$\mathbf{M}_{2}^{(d)} \equiv\left\{\mathbf{M}_{21}^{(d)}, \mathbf{M}_{22}^{(d)}\right\}$

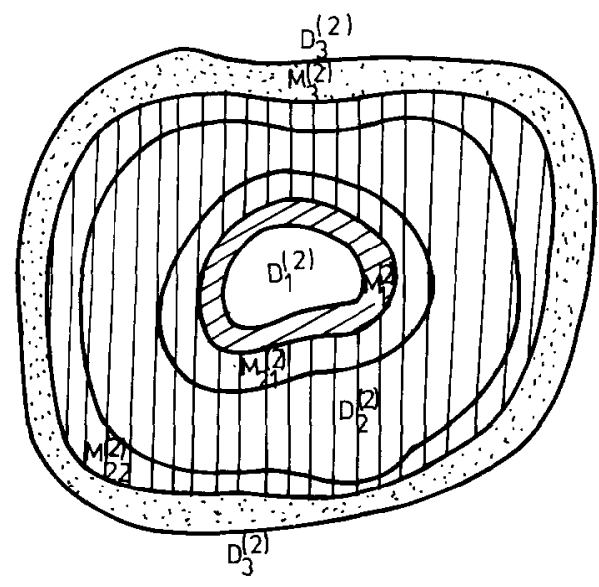

a

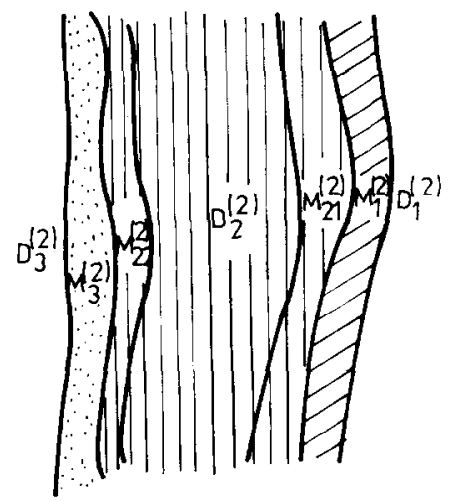

b

Fig. 2. Domains $D_{1}^{(2)}, D_{2}^{(2)}$ and $D_{3}^{(2)}$. The domain $D_{2}^{(2)}$ is bounded by two disconnected interfaces $\mathbf{M}_{21}^{(2)}$ and $\mathbf{M}_{22}^{(2)}$ in contact with the domains $D_{1}^{(2)}$ and $D_{2}^{(2)}$ bounded respectively by $\mathbf{M}_{1}^{(2)}$ and $\mathbf{M}_{3}^{(2)}$. (a) The case of a finite domain $D_{2}^{(2)}$ in contact with a finite domain $D_{1}^{(2)}$ and a semi-infinite one $\mathrm{D}_{3}^{(2)}$. (b) A slab domain $\mathrm{D}_{2}^{(2)}$ in contact with two semi-infinite domains $\mathrm{D}_{1}^{(2)}$ and $\mathrm{D}_{3}^{(2)}$. 
The cleavage which produces the two free surfaces bounding the system described in section 2.5.1 is done by an operator

$\boldsymbol{V}_{01}+\boldsymbol{V}_{02}$, defined in $\mathrm{D}_{\infty}^{(d)}$,

although it has non-zero elements only in the space of the interfaces.

$\boldsymbol{V}_{0 j^{\prime}}(j=1$ or 2$)$ is the cleavage operator which creates the $j$ free surface.

The operator $\boldsymbol{H}_{0}$ of this infinite system in $\mathrm{D}_{\infty}^{(d)}$ becomes $\boldsymbol{h}_{02}$ for the finite system described here, with

$\boldsymbol{h}_{02}=\boldsymbol{H}_{0}+\boldsymbol{V}_{01}+\boldsymbol{V}_{02}, \quad$ in $\mathrm{D}_{\infty}^{(d)}$.

\subsubsection{Two-surfaces response operators}

Then as in section 2.4, define

$$
\boldsymbol{A}_{02}=\left(\boldsymbol{V}_{01}+\boldsymbol{V}_{02}\right) \boldsymbol{G}_{0}, \text { in } \mathrm{D}_{\infty}^{(d)} \text {, }
$$

where $\boldsymbol{G}_{0}$ was defined by eq. (2.1).

Define also the response function $\boldsymbol{g}_{02}$ by

$\boldsymbol{h}_{02} \cdot \boldsymbol{g}_{02}=\boldsymbol{l}$, in $\mathrm{D}_{\infty}^{(d)}$.

These definitions lead also here to

$\boldsymbol{g}_{02}\left(\boldsymbol{I}+\boldsymbol{A}_{\mathrm{0} 2}\right)=\boldsymbol{G}_{0}$, in $\mathrm{D}_{2}^{(2)}$.

As in section 2.4, it is straightforward to see that in order to obtain the elements $\boldsymbol{g}_{\mathrm{s} 2}$ of $\boldsymbol{g}_{02}$ inside $\mathrm{D}_{2}^{(d)}$, one needs only to know the elements $\boldsymbol{G}_{\mathrm{s} 2}$ of $\boldsymbol{G}_{0}$ and $\boldsymbol{A}_{\mathrm{s} 2}$ of $\boldsymbol{A}_{02}$ inside $\mathrm{D}_{2}^{(d)}$. One can then rewrite eq. (2.16) as

$\boldsymbol{g}_{\mathrm{s} 2}\left(\boldsymbol{I}+\boldsymbol{A}_{\mathrm{s} 2}\right)=\boldsymbol{G}_{\mathrm{s} 2}, \quad$ in $\mathrm{D}_{2}^{(d)}$.

In what follows $\boldsymbol{A}_{\mathrm{s} 2}$ is called the two-surfaces response operator.

Note that $\boldsymbol{A}_{\mathrm{s} 2}$ has non-zero elements only between a point situated in $\mathbf{M}_{21}^{(d)}$ or $\mathrm{M}_{22}^{(d)}$ and any point situated in $\mathrm{D}_{2}^{(d)}$. Equivalently

$\boldsymbol{A}_{\mathrm{s} 2}\left(\boldsymbol{x}, \boldsymbol{x}^{\prime}\right) \equiv 0$, for $\boldsymbol{x} \notin \mathrm{M}_{2}^{(d)}$.

\subsubsection{J-surfaces response operators}

The above results of section 2.5 .2 can be easily generalized to a system with $J$ disconnected free surfaces.

Define

$\mathbf{M}_{2}^{(d)}=\left\{\mathbf{M}_{21}^{(d)}, \ldots, \mathbf{M}_{2 j}^{(d)}, \ldots, \mathbf{M}_{2 J}^{(d)}\right\}$

then the cleavage operator

$$
\sum_{j=1}^{J} \boldsymbol{V}_{0 j}, \quad \text { in } \mathrm{D}_{\infty}^{(\boldsymbol{d})} \text {. }
$$


Finally define the $J$-surfaces response operator $\boldsymbol{A}_{\mathrm{s} J}$ defined in $\mathrm{D}_{2}^{(d)}$, but constructed in $\mathrm{D}_{\infty}^{(d)}$ from

$\boldsymbol{A}_{0 J}=\left(\sum_{j=1}^{N} \boldsymbol{V}_{0 j}\right) \boldsymbol{G}_{0}$, in $\mathrm{D}_{\infty}^{(d)}$.

Note that

$\boldsymbol{A}_{\mathrm{s} J}\left(\boldsymbol{x}, \boldsymbol{x}^{\prime}\right) \equiv 0$, for $\boldsymbol{x} \notin \mathbf{M}_{2}^{(d)}$.

Finally the response operator $\boldsymbol{g}_{\mathrm{s} J}$ of this system can be obtained from

$\boldsymbol{g}_{\mathrm{s} J}\left(\boldsymbol{I}+\boldsymbol{A}_{\mathrm{s} J}\right)=\boldsymbol{G}_{\mathrm{s} J}, \quad$ in $\mathrm{D}_{2}^{(d)}$,

where

$\boldsymbol{G}_{\mathrm{s} J} \equiv\left\{\begin{array}{ll}\boldsymbol{G}_{0}, & \text { inside } \mathrm{D}_{2}^{(d)} \\ \mathbf{0}, & \text { outside } \mathrm{D}_{2}^{(d)}\end{array}\right.$.

\subsection{Interfaces between two different subsystems}

\subsubsection{Definitions}

In section 4 , the response operators for two independent and complementary in $D_{\infty}^{(d)}$ systems of the same nature were obtained. This procedure can, of course, be repeated for a system of a different nature.

Now replace in $\mathrm{D}_{3}^{(d)}$ the original physical system by a new one, but leaving them independent. In what follows, their respective operators will be labelled by the index $i=1$ or 3 . Define $(2 \times 2)$ block diagonal matrices between the domains $\mathrm{D}_{1}^{(d)}$ and $\mathrm{D}_{3}^{(d)}$ for all the operators of these two independent systems limited by their ideally cleaved surface

$$
\begin{aligned}
& \boldsymbol{h}_{\mathrm{s}}=\left(\begin{array}{cc}
\boldsymbol{h}_{\mathrm{s}}(11) & \mathbf{0} \\
0 & \boldsymbol{h}_{\mathrm{s}}(33)
\end{array}\right), \quad \boldsymbol{A}_{\mathrm{s}}=\left(\begin{array}{cc}
\boldsymbol{A}_{\mathrm{s}}(11) & \mathbf{0} \\
0 & \boldsymbol{A}_{\mathrm{s}}(33)
\end{array}\right), \\
& \boldsymbol{G}_{\mathrm{s}}=\left(\begin{array}{cc}
\boldsymbol{G}_{\mathrm{s}}(11) & 0 \\
0 & \boldsymbol{G}_{\mathrm{s}}(33)
\end{array}\right), \quad \boldsymbol{g}_{\mathrm{s}}=\left(\begin{array}{cc}
\boldsymbol{g}_{\mathrm{s}}(11) & \mathbf{0} \\
0 & \boldsymbol{g}_{\mathrm{s}}(33)
\end{array}\right), \quad \text { in } \mathrm{D}_{\infty}^{(\mathrm{d})} .
\end{aligned}
$$

The index $i=1$ or 3 refers to the corresponding operators defined in $\mathrm{D}_{i}^{(d)}$ in section 2.4 and 0 is the operator zero.

Note that

$\boldsymbol{h}_{\mathrm{s}} \cdot \boldsymbol{g}_{\mathrm{s}}=\boldsymbol{I}, \quad$ in $\mathrm{D}_{\infty}^{(d)}$,

$\boldsymbol{g}_{\mathrm{s}}\left(\boldsymbol{I}+\boldsymbol{A}_{\mathrm{s}}\right)=\boldsymbol{G}_{\mathrm{s}}, \quad$ in $\mathrm{D}_{\infty}^{(d)}$. 
Couple now these two different and independent systems by an operator $\boldsymbol{V}_{\mathrm{I}}$ which adds to $\boldsymbol{h}_{\mathrm{s}}$ and define it by

$\boldsymbol{h}_{\mathrm{I}}=\boldsymbol{h}_{\mathrm{s}}+\boldsymbol{V}_{\mathrm{I}}$, in $\mathrm{D}_{\infty}^{(d)}$.

\subsubsection{Interface response operators}

The corresponding resolvant operator $\boldsymbol{g}_{\mathrm{I}}$ is defined by

$\boldsymbol{h}_{\mathrm{I}} \cdot \boldsymbol{g}_{\mathrm{I}}=\boldsymbol{I}, \quad$ in $\mathrm{D}_{\infty}^{(d)}$.

From the above definitions, one ohtains at once

$\boldsymbol{g}_{\mathrm{I}}\left(\boldsymbol{I}+\boldsymbol{V}_{\mathrm{I}} \boldsymbol{g}_{\mathrm{s}}\right)=\boldsymbol{g}_{\mathrm{s}}, \quad$ in $\mathrm{D}_{\infty}^{(d)}$.

Multiply eq. (2.29) from the right by $\boldsymbol{I}+\boldsymbol{A}_{\mathrm{s}}$ and use eq. (2.26), to obtain

$\boldsymbol{g}_{\mathrm{I}}\left(\boldsymbol{I}+\mathbf{A}_{\mathrm{I}}\right)=\boldsymbol{G}_{\mathrm{s}}, \quad$ in $\mathrm{D}_{\infty}^{(d)}$,

where

$\boldsymbol{I}+\boldsymbol{A}_{\mathrm{I}}=\left(\boldsymbol{I}+\boldsymbol{V}_{\mathrm{I}} \boldsymbol{g}_{\mathrm{s}}\right)\left(\boldsymbol{I}+\boldsymbol{A}_{\mathrm{s}}\right)$, in $\mathrm{D}_{\infty}^{(d)}$.

Use eq. (2.26) to obtain

$\boldsymbol{A}_{\mathrm{I}}=\boldsymbol{A}_{\mathrm{s}}+\boldsymbol{V}_{\mathrm{I}} \boldsymbol{G}_{\mathrm{s}}$, in $\mathrm{D}_{\infty}^{(d)}$.

So the interface response operator $\boldsymbol{A}_{\mathrm{I}}$ is the linear superposition of the ideal surface response operator $\boldsymbol{A}_{\mathrm{s}}$ (eq. (2.24) and of the interface-suirface response operator

$\boldsymbol{A}_{\mathrm{Is}}=\boldsymbol{V}_{\mathrm{I}} \boldsymbol{G}_{\mathrm{s}}$, in $\mathrm{D}_{\infty}^{(d)}$.

Let us make more explicit this interface response operator $\boldsymbol{A}_{\mathrm{I}}$ by writing

$\boldsymbol{V}_{\mathrm{I}}=\left(\begin{array}{ll}\boldsymbol{V}_{\mathrm{I}}(11) & \boldsymbol{V}_{\mathrm{I}}(13) \\ \boldsymbol{V}_{\mathrm{I}}(31) & \boldsymbol{V}_{\mathrm{I}}(33)\end{array}\right), \quad$ in $\mathrm{D}_{\infty}^{(d)}$,

as a $(2 \times 2)$ matrix between the two subsystems. Then

$\boldsymbol{A}_{\mathrm{I}}=\left(\begin{array}{cc}\boldsymbol{A}_{\mathrm{s}}(11) & \mathbf{0} \\ \mathbf{0} & \boldsymbol{A}_{\mathrm{s}}(33)\end{array}\right)+\left(\begin{array}{ll}\boldsymbol{V}_{\mathrm{I}}(11) & \boldsymbol{V}_{\mathrm{I}}(13) \\ \boldsymbol{V}_{\mathrm{I}}(31) & \boldsymbol{V}_{\mathrm{I}}(33)\end{array}\right)\left(\begin{array}{cc}\boldsymbol{G}_{\mathrm{s}}(11) & \mathbf{0} \\ \mathbf{0} & \boldsymbol{G}_{\mathrm{s}}(33)\end{array}\right)$, in $\mathrm{D}^{(d)}$

Note that $\boldsymbol{V}_{\mathrm{I}}$ has non-zero elements only in the space of

$\mathbf{M}^{(d)} \equiv\left\{\mathbf{M}_{1}^{(d)}, \mathbf{M}_{3}^{(d)}\right\}$,

and that

$\boldsymbol{A}_{\mathrm{I}}\left(\boldsymbol{x}, \boldsymbol{x}^{\prime}\right) \equiv 0$, for $\boldsymbol{x} \notin \mathrm{M}^{(\mathrm{d})}$.

The extension in space, respectively $\mathbf{M}_{1}^{(d)}$ and $\mathbf{M}_{2}^{(d)}$ of the cleavage operators which created the two independent subsystems may be smaller than 
the extension in space of the coupling operator $\boldsymbol{V}_{\mathrm{I}}$. Then one has to redefine $\mathbf{M}_{1}^{(d)}$ and $\mathbf{M}_{2}^{(d)}$, in agreement with the largest extension and the above result still holds.

\subsection{3. $J$ interfaces between two different subsystems}

The results of section 2.6 .2 can be easily generalized to a system with $J$ disconnected interfaces between two different subsystems.

Define

$\mathbf{M}_{i}^{(d)}=\left\{\mathbf{M}_{i 1}^{(d)}, \ldots, \mathbf{M}_{i j}^{(d)}, \ldots, \mathbf{M}_{i J}^{(d)}\right\}, \quad i=1$ or 3,

and the coupling operator

$\boldsymbol{V}_{\mathrm{I} J}=\sum_{j=1}^{J} \boldsymbol{V}_{\mathrm{I} J}$, in $\mathrm{D}_{\infty}^{(d)}$.

Define also the $J$-interfaces response operator $\boldsymbol{A}_{I J}$, defined in $D_{\infty}^{(d)}$ as

$\boldsymbol{A}_{\mathrm{I} J}=\boldsymbol{A}_{\mathrm{s} 2 J}+\boldsymbol{V}_{\mathrm{I} J} \boldsymbol{G}_{\mathrm{s} 2 J}$, in $\mathrm{D}_{\infty}^{(d)}$,

where $\boldsymbol{A}_{\mathrm{s} J}$ is the $2 J$-surfaces response operator defined for the $J$ disconnected surfaces of each subsystem like in section 2.5.2 and $\boldsymbol{G}_{\mathrm{s} 2 J}$ is the corresponding hulk response operator of both subsystems without interactions through their $J$ interfaces. Note that

$\boldsymbol{A}_{\mathrm{I} J}\left(\boldsymbol{x}, \boldsymbol{x}^{\prime}\right) \equiv 0$, for $\boldsymbol{x} \notin \mathrm{M}^{(d)}$.

Finally the response operator $\boldsymbol{g}_{\mathrm{I} J}$ of this system with $J$ interfaces can be obtained from

$\boldsymbol{g}_{\mathrm{I} J}\left(\boldsymbol{I}+\boldsymbol{A}_{\mathrm{I} J}\right)=\boldsymbol{G}_{\mathrm{s} 2 J}$, in $\mathrm{D}_{\infty}^{(d)}$.

\subsection{One adsorbed system on another one}

\subsubsection{Definitions}

Couple the system with two disconnected free surfaces defined in section 5 , to the finite or semi-infinite complementary-in-space surface system defined in section 4 . We create in this manner (see fig. 2) cithcr a coupled finite system with another finite one adsorbed on it in $\left\{\mathrm{D}_{1}^{(d)}, \mathrm{D}_{2}^{(d)}\right\}$ or a semi-infinite system with an adsorbed finite one adsorbed on it in $\left\{\mathrm{D}_{2}^{(d)}, \mathrm{D}_{3}^{(d)}\right\}$. In both cases the adsorbate has a free surface in contact with the vacuum. The case of an open interface (fig. 2b) between these two subsystems is a simple example of a semi-infinite system with an adsorbate.

Before switching on the coupling between these two independent systems, define $(2 \times 2)$ block diagonal matrices between the adsorbate and the sub- 
strate, in the same manner as in section 2.6.1 and inside

$\mathrm{D}^{(d)} \equiv\left\{\mathrm{D}_{2}^{(d)}, \mathrm{D}_{i}^{(d)}\right\}, \quad i=1$ or 3 ,

$\begin{array}{ll}\boldsymbol{h}_{\mathrm{s} 3}=\left(\begin{array}{cc}\boldsymbol{h}_{\mathrm{s} 2}(22) & \mathbf{0} \\ \mathbf{0} & \boldsymbol{h}_{\mathrm{s}}(i i)\end{array}\right), & \boldsymbol{A}_{\mathrm{s} 3}=\left(\begin{array}{cc}\boldsymbol{A}_{\mathrm{s} 2}(22) & \mathbf{0} \\ \mathbf{0} & \boldsymbol{A}_{\mathrm{s}}(i i)\end{array}\right), \\ \boldsymbol{G}_{\mathrm{s} 3}=\left(\begin{array}{cc}\boldsymbol{G}_{\mathrm{s} 2}(22) & \mathbf{0} \\ \mathbf{0} & \boldsymbol{G}_{\mathrm{s}}(\mathrm{ii})\end{array}\right), & \boldsymbol{g}_{\mathrm{s} 3}=\left(\begin{array}{cc}\boldsymbol{g}_{\mathrm{s} 2}(22) & \mathbf{0} \\ \mathbf{0} & \boldsymbol{g}_{\mathrm{s}}(i i)\end{array}\right), \quad \text { in } \mathrm{D}^{(d)}, i=1 \text { or } 3 .\end{array}$

The indices $(22)$ or $(i i)=(11)$ or $(33)$ refer to the corresponding operators defined respectively in $\mathrm{D}_{2}^{(d)}$ (section 2.5) and in $\mathrm{D}_{i}^{(d)}$ (section 2.4).

Note that

$\boldsymbol{h}_{\mathrm{s} 3} \cdot \boldsymbol{g}_{33}=\boldsymbol{l}, \quad$ in $\mathrm{D}^{(d)}$,

$\boldsymbol{g}_{\mathrm{s} 3}\left(\boldsymbol{I}+\boldsymbol{A}_{\mathrm{s} 3}\right)=\boldsymbol{G}_{\mathrm{s}}$, in $\mathrm{D}^{(d)}$.

Couple now these two different and independent systems by an operator $\boldsymbol{V}_{\mathrm{I}}$, defined in

$\left\{\mathbf{M}_{21}^{(d)}, \mathbf{M}_{1}^{(d)}\right\}$ or $\left\{\mathbf{M}_{22}^{(d)}, \mathbf{M}_{3}^{(d)}\right\}$,

depending on whether the adsorbate is coupled with the finite $\mathrm{D}_{1}^{(d)}$ system or with the semi-infinite $\mathrm{D}_{3}^{(d)}$ one.

The operator of interest in this section will be

$\boldsymbol{h}_{\mathrm{A}}=\boldsymbol{h}_{\mathrm{s} 3}+\boldsymbol{V}_{\mathrm{I}}$, in $\mathrm{D}^{(d)}$.

\subsubsection{Response operators}

The resolvant operator $\boldsymbol{g}_{\mathrm{A}}$ is defined here also as

$\boldsymbol{h}_{\mathrm{A}} \cdot \boldsymbol{g}_{\mathrm{A}}=\boldsymbol{I}$, in $\mathrm{D}^{(d)}$.

From the above definitions, it follows at once that

$\boldsymbol{g}_{\mathrm{A}}\left(\boldsymbol{I}+\boldsymbol{V}_{\mathrm{I}} \boldsymbol{g}_{\mathrm{s} 3}\right)=\boldsymbol{g}_{\mathrm{s} 3}, \quad$ in $\mathrm{D}^{(d)}$.

Multiply this equation from the right by $\boldsymbol{I}+\mathbf{A}_{\mathrm{s} 3}$ and use eq. (2.45) to obtain $\boldsymbol{g}_{\mathrm{A}} \cdot\left(\boldsymbol{I}+\boldsymbol{A}_{\mathrm{A}}\right)=\boldsymbol{G}_{\mathrm{s} 3}$, in $\mathrm{D}^{(d)}$,

where the adsorbate response operator $\boldsymbol{A}_{\mathrm{A}}$ is given by

$\boldsymbol{A}_{\mathrm{A}}=\boldsymbol{A}_{\mathrm{s}}+\boldsymbol{V}_{\mathrm{I}} \boldsymbol{G}_{\mathrm{s}}$, in $\mathrm{D}^{(d)}$.

$\boldsymbol{V}_{I} \boldsymbol{G}_{s}$ will be called the ideal surface adsorbate response operator.

Let us work out a more explicit expression of $\boldsymbol{A}_{\mathrm{A}}$ in the form of $(2 \times 2)$ matrices between the two different subsystems

$$
\begin{aligned}
& \boldsymbol{A}_{\mathrm{A}}=\left(\begin{array}{cc}
\boldsymbol{A}_{\mathrm{s} 2}(22) & \mathbf{0} \\
\mathbf{0} & \boldsymbol{A}_{\mathrm{s}}(i i)
\end{array}\right)+\left(\begin{array}{ll}
\boldsymbol{V}_{\mathrm{I}}(22) & \boldsymbol{V}_{\mathrm{I}}(2 i) \\
\boldsymbol{V}_{\mathrm{I}}(i 2) & \boldsymbol{V}_{\mathrm{I}}(i i)
\end{array}\right)\left(\begin{array}{cc}
\boldsymbol{G}_{\mathrm{s} 2}(22) & \mathbf{0} \\
\mathbf{0} & \boldsymbol{G}_{\mathrm{s}}(i i)
\end{array}\right), \\
& \quad \text { in } \mathrm{D}^{(d)}
\end{aligned}
$$


where the subscript $s 2$ reminds one of the fact that the adsorbate had two disconnected ideal surfaces.

Note that

$\boldsymbol{A}_{\mathrm{A}}\left(\boldsymbol{x}, \boldsymbol{x}^{\prime}\right) \equiv 0$, for $\boldsymbol{x} \notin \mathrm{M}^{\left(d^{\prime}\right)}$,

where

$\mathbf{M}^{(d)} \equiv\left\{\mathbf{M}_{21}^{(d)}, \mathbf{M}_{22}^{(d)}, \mathbf{M}_{i}^{(d)}\right\}, \quad i=1$ or 3

\subsubsection{Disconnected adsorptions of one subsystem on another one}

In the same manner as in sections 2.5 .3 and 2.6.3, one can write down easily the generalization of the results of section 2.7.2 to $J$ disconnected adsorptions of one subsystem on another one. Let us distinguish here two cases.

2.7.3.1. Adsorption on $K$ disconnected voids. Inside the infinite $\mathrm{D}_{\infty}^{(d)}$ space, the system 3 may have $K$ disconnected voids on which may be adsorbed the same adsorbate 2 .

Define

$$
\begin{aligned}
& \mathbf{M}_{2 j}^{(d)}=\left\{\mathbf{M}_{2 j 1}^{(d)}, \ldots, \mathbf{M}_{2 j k}^{(d)}, \ldots, \mathbf{M}_{2 j K}^{(d)}\right\}, \quad j=1 \text { or } 2, \\
& M_{3}^{(d)}=\left\{\mathbf{M}_{31}^{(d)}, \ldots, \mathbf{M}_{3 k}^{(d)}, \ldots, \mathbf{M}_{3 K}^{(d)}\right\},
\end{aligned}
$$

and the coupling operator

$$
\boldsymbol{V}_{\mathrm{I} K}=\sum_{k=1}^{K} \boldsymbol{V}_{\mathrm{I} k}, \quad \text { in } \mathrm{D}^{(d)} \text {. }
$$

The $K$ adsorption response operator $\boldsymbol{A}_{\mathrm{A} K}$ is then defined in $\mathrm{D}^{(d)}$ by

$\boldsymbol{A}_{\mathrm{A} K}=\boldsymbol{A}_{\mathrm{s} 3 K}+\boldsymbol{V}_{\mathrm{I} K} \boldsymbol{G}_{\mathrm{s} 3 K}, \quad$ in $\mathrm{D}^{(d)}$.

This is an easy generalization of eq. (2.39). Here also as in eqs. (2.52)

$\boldsymbol{A}_{\mathrm{A} K}\left(\boldsymbol{x}, \boldsymbol{x}^{\prime}\right) \equiv 0$, for $\boldsymbol{x} \notin \mathrm{M}^{(d)}$.

And the response operator $\boldsymbol{g}_{\mathrm{A} K}$ for this system with adsorption of $K$ disconnected voids is given by

$\boldsymbol{g}_{\mathrm{A} K}\left(\boldsymbol{I}+\boldsymbol{A}_{\mathrm{A} K}\right)=\boldsymbol{G}_{\mathrm{s} 3 K}$, in $\mathrm{D}^{(d)}$.

2.7.3.2. Adsorption of $K$ clusters. On the surface of the finite $\mathrm{D}_{1}^{(d)}$ system or of the semi-infinite $D_{3}^{(d)}$ system it is possible to adsorb one or several finite clusters of another system. These finite clusters have the same properties as the $\mathrm{D}_{1}^{(d)}$ system, but will be called here $\mathrm{D}_{4 k}^{(d)}$ such that

$\mathrm{D}_{4}^{(d)} \equiv\left\{\mathrm{D}_{41}^{(d)}, \ldots, \mathrm{D}_{4 k}^{(d)}, \ldots, \mathrm{D}_{4 K}^{(d)}\right\}$ 
These clusters when adsorbed on the $\mathrm{D}_{i}^{(d)}$ system, $i=1$ or 3 , will have with this system an interface of contact

$\mathbf{M}_{4}^{(d)} \equiv\left\{\mathbf{M}_{41}^{(d)}, \ldots, \mathbf{M}_{4 k}^{(d)}, \ldots, \mathbf{M}_{4 K}^{(d)}\right\}$

and

$\mathbf{M}_{i}^{(d)} \equiv\left\{M_{i 1}^{(d)}, \ldots, \mathbf{M}_{i k}^{(d)}, \ldots, \mathbf{M}_{i K}^{(d)}\right\}, \quad i=1$ or 3.

Define here also the corresponding coupling operator

$\boldsymbol{V}_{1 K}=\sum_{k=1}^{K} \boldsymbol{V}_{\mathrm{I} K}, \quad$ in $\mathrm{D}^{(d)}$

and the $K$ clusters response operator

$\boldsymbol{A}_{\mathrm{c} K}=\boldsymbol{A}_{\mathrm{s} 2 K}+\boldsymbol{V}_{\mathrm{I} K} \boldsymbol{G}_{\mathrm{s} 2 K}, \quad$ in $\mathrm{D}^{(d)}$,

where $\boldsymbol{A}_{\mathrm{s} 2 K}$ and $\boldsymbol{G}_{\mathrm{s} 2 K}$ are defined as in section 2.6.3.

The response operator $g_{\mathrm{c} K}$ of this system with $K$ adsorbed clusters will here also be given by

$\boldsymbol{g}_{\mathrm{c} K}\left(\boldsymbol{I}+\boldsymbol{A}_{\mathrm{c} K}\right)=\boldsymbol{G}_{\mathrm{s} 2 K}, \quad$ in $\mathrm{D}^{(d)}$.

\subsection{One membrane between two different systems}

\subsubsection{Definitions}

We couple the system with two disconnected free surfaces defined in section 2.5 , to the two complementary in $D_{\infty}^{(d)}$ finite and semi-infinite different systems defined in section 2.4. See fig. 2 for an illustration in two dimensions.

This way we create one finite $\mathrm{D}_{2}^{(d)}$ membrane separating the finite (or semi-infinite) $\mathrm{D}_{1}^{(d)}$ system from the semi-infinite $\mathrm{D}_{3}^{(d)}$ one.

Before switching on the coupling between these three independent systems, let us define $(3 \times 3)$ block diagonal matrices between these three $\mathrm{D}_{i}^{(d)}(i=$ $1,2,3)$ domains

$$
\begin{aligned}
& \boldsymbol{h}_{\mathrm{s} 4}=\left(\begin{array}{ccc}
\boldsymbol{h}_{\mathrm{s}}(11) & \mathbf{0} & \mathbf{0} \\
\mathbf{0} & \boldsymbol{h}_{\mathrm{s} 2}(22) & \mathbf{0} \\
\mathbf{0} & \mathbf{0} & \boldsymbol{h}_{\mathrm{s}}(33)
\end{array}\right), \text { in } \mathrm{D}_{\infty}^{(d)}, \\
& \boldsymbol{G}_{\mathrm{s} 4}=\left(\begin{array}{ccc}
\boldsymbol{G}_{\mathrm{s}}(11) & \mathbf{0} & \mathbf{0} \\
\mathbf{0} & \boldsymbol{G}_{\mathrm{s} 2}(22) & \mathbf{0} \\
\mathbf{0} & \mathbf{0} & \boldsymbol{G}_{\mathrm{s}}(33)
\end{array}\right), \text { in } \mathrm{D}_{\infty}^{(d)}, \\
& \boldsymbol{A}_{\mathrm{s} 4}=\left(\begin{array}{ccc}
\boldsymbol{A}_{\mathrm{s}}(11) & \mathbf{0} & \mathbf{0} \\
\mathbf{0} & \boldsymbol{A}_{\mathrm{s} 2}(22) & \mathbf{0} \\
\mathbf{0} & \mathbf{0} & \boldsymbol{A}_{\mathrm{s}}(33)
\end{array}\right), \text { in } \mathrm{D}_{\infty}^{(d)},
\end{aligned}
$$


$\boldsymbol{g}_{s 4}=\left(\begin{array}{ccc}\boldsymbol{g}_{\mathrm{s}}(11) & \mathbf{0} & \mathbf{0} \\ \mathbf{0} & \boldsymbol{g}_{\mathrm{s} 2}(22) & \mathbf{0} \\ \mathbf{0} & \mathbf{0} & \boldsymbol{g}_{\mathrm{s}}(33)\end{array}\right), \quad$ in $\mathrm{D}_{\infty}^{(d)}$,

where the $\boldsymbol{h}_{\mathrm{s}}(i i), \boldsymbol{G}_{\mathrm{s}}(i i), \boldsymbol{A}_{\mathrm{s}}(i i)$ and $\boldsymbol{g}_{\mathrm{s}}(i i)$ were obtained for $i=1$ and 3 in section 2.4 and the $\boldsymbol{h}_{\mathrm{s} 2}(22), \boldsymbol{g}_{22}(22), \boldsymbol{A}_{\mathrm{s} 2}(22)$ and $\boldsymbol{g}_{\mathrm{s} 2}(22)$ in section 2.5 .

Note also that

$$
\begin{array}{ll}
\boldsymbol{h}_{\mathrm{s} 4} \cdot \boldsymbol{g}_{\mathrm{s4}}=\boldsymbol{I}, & \text { in } \mathrm{D}_{\infty}^{(d)}, \\
\boldsymbol{g}_{\mathrm{s4}}\left(\boldsymbol{I}+\boldsymbol{A}_{\mathrm{s} 4}\right)=\boldsymbol{G}_{\mathrm{s4}}, & \text { in } \mathrm{D}_{\infty}^{(d)} .
\end{array}
$$

Couple now these three different and independent systems by an operator $\boldsymbol{V}_{\mathbf{M}}$, defined inside the two interfaces

$\left\{\mathbf{M}_{1}^{(d)}, \mathbf{M}_{21}^{(d)}\right\}$,

$\left\{\mathbf{M}_{22}^{(d)}, \mathbf{M}_{3}^{(d)}\right\}$.

The operator of interest here will therefore be

$\boldsymbol{h}_{\mathrm{M}}=\boldsymbol{h}_{\mathrm{s} 4}+\boldsymbol{V}_{\mathrm{M}}$, in $\mathrm{D}_{\infty}^{(d)}$,

with

$\boldsymbol{V}_{M}=\left(\begin{array}{ccc}\boldsymbol{V}_{M}(1,1) & \boldsymbol{V}_{M}(1,2) & 0 \\ \boldsymbol{V}_{M}(2,1) & \boldsymbol{V}_{M}(2,2) & \boldsymbol{V}_{M}(2,3) \\ 0 & \boldsymbol{V}_{M}(3,2) & \boldsymbol{V}_{M}(3,3)\end{array}\right), \quad$ in $D_{\infty}^{(d)}$

We supposed that their is no direct coupling between the systems $D_{1}^{(d)}$ and $\mathrm{D}_{3}^{(d)}$. However, if such a direct coupling would exist in some special cases, then all what follows can be easily generalized by introducing the $V_{M}(13)$ and $V_{M}(31)$ elements in (2.69).

\subsubsection{Membrane response operators}

The resolvant operator $\boldsymbol{g}_{\mathrm{M}}$ is defined here also as

$\boldsymbol{h}_{\mathrm{M}} \cdot \boldsymbol{g}_{\mathrm{m}}=\boldsymbol{l}$, in $\mathrm{D}_{\infty}^{(d)}$.

From the above definitions, it is straightforward to show that

$\boldsymbol{g}_{\mathrm{M}}\left(\boldsymbol{l}+\boldsymbol{V}_{\mathrm{M}} \boldsymbol{g}_{\mathrm{s} 4}\right)=\boldsymbol{g}_{\mathrm{s} 4}$, in $\mathrm{D}_{\infty}^{(d)}$.

Multiply eq. (2.71) from the right by $\boldsymbol{I}+\boldsymbol{A}_{\mathrm{s} 4}$ and use eq. (2.65) to show that $\boldsymbol{g}_{\mathrm{M}}\left(\boldsymbol{I}+\boldsymbol{A}_{\mathrm{M}}\right)=\boldsymbol{G}_{\mathrm{s} 4}$, in $\mathrm{D}_{\infty}^{(d)}$,

where the membrane response operator is

$A_{\mathrm{M}}=A_{s 4}+\boldsymbol{V}_{\mathrm{M}} \boldsymbol{G}_{\mathrm{s} 4}$, in $\mathrm{D}_{\infty}^{(d)}$.

Call $\boldsymbol{V}_{\mathrm{M}} \boldsymbol{G}_{\mathrm{s} 4}$ the membrane-two-surfaces response operator. 
Let us write out a more explicit form of $\boldsymbol{A}_{\mathrm{M}}$

$$
\begin{aligned}
\boldsymbol{A}_{\mathrm{M}}= & \left(\begin{array}{ccc}
\boldsymbol{A}_{\mathrm{s}}(11) & \mathbf{0} & \mathbf{0} \\
\mathbf{0} & \boldsymbol{A}_{\mathrm{s} 2}(22) & \mathbf{0} \\
\mathbf{0} & \mathbf{0} & \boldsymbol{A}_{\mathrm{s}}(33)
\end{array}\right) \\
& +\left(\begin{array}{ccc}
\boldsymbol{V}_{\mathrm{M}}(11) & \boldsymbol{V}_{\mathrm{M}}(12) & \mathbf{0} \\
\boldsymbol{V}_{\mathrm{M}}(21) & \boldsymbol{V}_{\mathrm{M}}(22) & \boldsymbol{V}_{\mathrm{M}}(23) \\
\mathbf{0} & \boldsymbol{V}_{\mathrm{M}}(32) & \mathbf{0}
\end{array}\right)\left(\begin{array}{ccc}
\boldsymbol{G}_{\mathrm{s}}(11) & \mathbf{0} & \mathbf{0} \\
\mathbf{0} & \boldsymbol{G}_{\mathrm{s} 2}(22) & \mathbf{0} \\
\mathbf{0} & \mathbf{0} & \boldsymbol{G}_{\mathrm{s}}(33)
\end{array}\right),
\end{aligned}
$$

in $\mathrm{D}^{(d)}$,

where the subscript $s 2$ reminds us that we build up the membrane from a system with two disconnected ideal surfaces. Note that

$\boldsymbol{A}_{\mathrm{M}}\left(\boldsymbol{x}, \boldsymbol{x}^{\prime}\right) \equiv 0$, for $\boldsymbol{x} \notin \mathrm{M}^{(d)}$.

\subsection{3. $K$ disconnected membranes}

As in sections $2.5 .3,2.6 .3$ and 2.7 .3 , it is easy to generalize the results of section 2.8 .2 to a system with $K$ disconnected membranes of one system

$\mathrm{D}_{2}^{(d)} \equiv\left\{\mathrm{D}_{21}^{(d)}, \ldots, \mathrm{D}_{2 k}^{(d)}, \ldots, \mathrm{D}_{2 K}^{(d)}\right\}$

separating finite pieces of another system

$\mathrm{D}_{1}^{(d)} \equiv\left\{\mathrm{D}_{11}^{(d)}, \ldots, \mathrm{D}_{1 k}^{(d)}, \ldots, \mathrm{D}_{1 K}^{(d)}\right\}$

from a third system $D_{3}^{(d)}$.

Here it is also possible to define a $K$ membranes response operator

$\boldsymbol{A}_{\mathrm{M} K}=\boldsymbol{A}_{\mathrm{s} 4 K}+\boldsymbol{V}_{\mathrm{MK}} \boldsymbol{G}_{\mathrm{s} 4 K}$,

in the same manner as in eqs. (2.39), (2.56) and (2.62).

The response function $\boldsymbol{g}_{\mathrm{MK}}$ of this system with $K$ disconnected membranes is given by

$\boldsymbol{g}_{\mathrm{MK}}\left(\boldsymbol{I}+\mathbf{A}_{\mathrm{MK}}\right)=\boldsymbol{G}_{\mathrm{s} 4 K}$,

where

$\boldsymbol{A}_{\mathrm{M} K}\left(\boldsymbol{x}, \boldsymbol{x}^{\prime}\right) \equiv 0$, for $\boldsymbol{x} \notin \mathrm{M}^{(d)}$,

$\mathbf{M}^{(d)}$ being the space of all interfaces.

\subsection{Any real composite system}

Any real composite system $\mathrm{D}^{(d)}$ formed out of $N$ different subsystems $\mathrm{D}_{i}^{(d)}$, $1 \leqq i \leqq N$, can be constructed out of the different systems defined in sections 2.4 to 2.8. Let us define the domains of existence of this system, of its 
subsystems and of its interfaces $\mathbf{M}_{i}^{(d)}$ and subinterfaces $\mathbf{M}_{i j}^{(d)}$. Call $\mathbf{M}^{(d)}$ the domain of all interfaces.

Construct as $(N \times N)$ matrices: $\boldsymbol{A}_{s N}$ the response of all ideal surfaces of all non-interacting subsystems; $\boldsymbol{V}_{I}$ the operator, which couples these non-interacting subsystems and $\boldsymbol{G} \equiv \boldsymbol{G}_{s N}$ the bulk response of all these subsystems but contained inside their domain of existence. Then define the response of all interfaces and surfaces of the real composite system under study as

$\boldsymbol{A}=\boldsymbol{A}_{\mathrm{s} N}+\boldsymbol{V}_{\mathrm{I}} \boldsymbol{G}$, in $\mathrm{D}^{(d)}$.

It is obvious from the considerations developed above that the response operator $\boldsymbol{g}$ of this system can be obtained from

$\boldsymbol{g} \cdot(\boldsymbol{I}+\boldsymbol{A})=\boldsymbol{G}$, in $\mathrm{D}^{(d)}$.

Note also that the interface response operator $\boldsymbol{A}$, is such that

$\boldsymbol{A}\left(\boldsymbol{x}, \boldsymbol{x}^{\prime}\right) \equiv 0$, for $\boldsymbol{x} \notin \mathrm{M}^{(d)}$.

Thus we showed that the response operator $\boldsymbol{g}$ of any real composite system is given by the general equation (2.81), as a function of the bulk responses $\boldsymbol{G}$ defined inside the domain of existence of each subsystem and of an interface response operator $\boldsymbol{A}$. This operator $\boldsymbol{A}$ can be easily constructed as a linear superposition of the ideal surface response opcrator $\boldsymbol{A}_{\mathrm{s}_{N}}$ of all non-interacting subsystems and of the surface-interface response operator $\boldsymbol{V}_{\mathrm{I}} \boldsymbol{G}$ (eq. (2.81)).

\subsection{Different methods for including defects in a real system}

\subsubsection{The bulk-surface method for defects}

All defects in any real composite system can be included into the above theory in the manner described above. A few examples will illustrate this.

An atomic vacancy in a solid can be studied as an ideally truncated internal surface as described in section 2.4 on which one adsorbs a few shells of atoms with perturbed properties (section 2.7).

A substitutional impurity in a solid can be studied as an interface between two different systems (section 2.6).

An interstitial or in general an intercalated atom, compound or layer can also be treated in the above manner. One would first have to define fictitious internal free surfaces in the system and then introduce into these voids the intercalated system.

The substitutional impurities and the intercalated systems can be cut out of the corresponding infinite systems, in order to have in $\boldsymbol{G}$ the bulk response function of these infinite systems defined inside their domain.

The real free surface can also be constructed as an ideally cleaved free surface on which one adsorbs a few perturbed layers. 
Dislocations, grain boundaries,..., any defect of any dimensions can be modelled along the lines described above.

The method proposed until now seems certainly to be the easiest way to obtain response functions for real composite systems constructed out of important pieces of bulk materials. However, the few examples of this section suggest that this bulk-surface method for defects can be improved when one has to deal with small dimensional defects.

\subsubsection{The surface method for defects}

In many cases, it is easier to calculate the response function $\boldsymbol{g}_{\mathrm{s}}$ (ii) of a finite subsystem $D_{i}^{(d)}$ with ideal surfaces, rather than the bulk response function $\mathbf{G}_{\mathrm{s}}(i i)$ of the corresponding infinite system. This is true for one atom or a small cluster of atoms, one atomic layer or a slab of a few atomic layers, for example.

Then it is more convenient to leave in the reference block diagonal response function $\boldsymbol{G}$, this $\boldsymbol{g}_{\mathrm{s}}(i i)$ rather than to use eq. (2.8) in order to eliminate $\boldsymbol{g}_{\mathrm{s}}(i i)$ to the benefit of $\boldsymbol{G}_{\mathrm{s}}(\mathrm{ii})$.

It is straightforward to see that the general equations (2.81) and (2.82) remain valid in this surface method for defects, once one includes in eq. (2.81)

$\mathbf{A}_{\mathrm{s}}(i i)=0, \quad$ in $\mathbf{M}_{i}^{(d)}$

and in $\boldsymbol{G}$, the block $\boldsymbol{g}_{\mathrm{s}}(i i)$ rather than the block $\boldsymbol{G}_{\mathrm{s}}(i i)$.

\subsubsection{The perturbed bulk method for defects in composite systems}

Often some properties of the operator $\boldsymbol{h}$ of a real system are perturbed. This is for example the case of the interactions near localized defects, which can be described by assuming that the defects are due to modifications of the properties of the system. It is indeed the classical Dyson way for calculating the response function of a system perturbed by a localized defect. Let us call $\boldsymbol{V}_{p}$ this perturbation of $\boldsymbol{h}$ and note that $\boldsymbol{V}_{\mathrm{p}}$ can be the sum of several perturbations affecting any part of the domain $\mathrm{D}^{(d)}$, including its interface domain $\mathrm{M}^{(d)}$. We write

$\boldsymbol{h}_{\mathrm{p}}=\boldsymbol{h}+\mathbf{V}_{\mathrm{p}}$.

Due to this perturbation $\boldsymbol{V}_{\mathrm{p}}$, the response function $\boldsymbol{g}$ will be changed into a new response function $\boldsymbol{g}_{\mathrm{p}}$ such that

$\boldsymbol{g}_{\mathrm{p}}\left(\boldsymbol{I}+\boldsymbol{V}_{\mathrm{p}} \boldsymbol{g}\right)=\boldsymbol{g}$.

Multiply this equation from the right by $I+\boldsymbol{A}$ and use eq. (2.81) to obtain $\boldsymbol{g}_{\mathrm{P}}\left(\boldsymbol{I}+\boldsymbol{A}_{\mathrm{p}}\right)=\boldsymbol{G}$,

with

$$
A_{p}=A+V_{p} G \text {. }
$$


These results show that the general equation (2.82) remains valid for this treatment of perturbative defects, when redefining the general interface responses $A$ of eq. (2.81) as

$\boldsymbol{A}=\boldsymbol{A}_{\mathrm{s} N}+\left(\boldsymbol{V}_{\mathrm{I}}+\boldsymbol{V}_{\mathrm{p}}\right) \boldsymbol{G}$, in $\mathrm{D}^{(d)}$.

Note that the interface domain $\mathbf{M}^{(d)}$ is increased by the domains affected by the perturbations $V_{p}$.

Remember that $\boldsymbol{V}_{\mathrm{p}}$ was defined (eq. (2.85)) as the perturbation of $\boldsymbol{h}$. If these defects affect only the bulk properties of each subsystem then $\boldsymbol{V}_{\mathrm{p}}$ perturbs only the $\sum_{i=1}^{N} \boldsymbol{H}_{0}(i i)$, but, in general $\boldsymbol{V}_{\mathrm{p}}$ can also be a perturbation of the interface domain $\mathbf{M}^{(d)}$.

When is it better to use for the study of defects in real systems this perturbed bulk method rather than the ones given before? It is advantageous to use in each specific case the one which gives the simplest calculations. In particular it is clear that when only the properties of one atom or of one translationally invariant atomic layer are perturbed, then the method of this section should be the simplest one.

\subsection{The alternative general equation}

Instead of defining the response functions like in eqs. (2.1) and (2.3), one may define them equivalently as

$$
\begin{array}{ll}
\boldsymbol{G}_{0} \cdot \boldsymbol{H}_{0}=\boldsymbol{I}, & \text { in } \mathrm{D}_{\infty}^{(d)}, \\
\boldsymbol{g}_{0} \cdot \boldsymbol{h}_{0}=\boldsymbol{I}, & \text { in } \mathrm{D}_{\infty}^{(d)} .
\end{array}
$$

Then eqs. (2.4) and (2.5) can be written equivalently as

$$
\begin{array}{ll}
\boldsymbol{A}_{0}^{\prime}=\boldsymbol{G}_{0} \boldsymbol{V}_{0}, & \text { in } \mathrm{D}_{\infty}^{(d)}, \\
\left(\boldsymbol{I}+\boldsymbol{A}_{0}^{\prime}\right) \boldsymbol{g}_{0}=\boldsymbol{G}_{0}, & \text { in } \mathrm{D}_{\infty}^{(d)} .
\end{array}
$$

And with the help of eq. (2.7), one can also write

$$
\left(\boldsymbol{I}+\boldsymbol{A}_{\mathrm{s}}^{\prime}\right) \boldsymbol{g}_{\mathrm{s}}=\boldsymbol{G}_{\mathrm{s}}, \quad \text { in } \mathrm{D}_{i}^{(d)}, i=1 \text { or } 3,
$$

as an equivalent symmetrical form of eq. (2.8) giving the response $\boldsymbol{g}_{\mathrm{s}}$ of an ideal finite or semi-infinite system as a function of $\boldsymbol{G}_{\mathrm{s}}$ and $\boldsymbol{A}_{\mathrm{s}}^{\prime}$. Recall that $\boldsymbol{G}_{\mathrm{s}}$ is the bulk response function confined inside $D_{i}^{(d)}$. The equivalent surface response operator $\boldsymbol{A}_{s}^{\prime}$ has also non-zero elements only inside $\mathrm{D}_{i}^{(d)}$, but these elements have to be calculated from eq. (2.91a) in $\mathrm{D}_{\infty}^{(d)}$.

Note that

$$
\boldsymbol{A}_{\mathrm{s}}^{\prime}\left(\boldsymbol{x}, \boldsymbol{x}^{\prime}\right) \equiv 0, \text { for } \quad \boldsymbol{x}^{\prime} \notin \mathrm{M}_{\mathrm{i}}^{(d)} \text {. }
$$

All the above results for systems bounded by one or several surfaces can be rewritten in the same manner and especially eqs. (2.17) and (2.22). 
It is also straightforward to show that eq. (2.30) for a system with one interface can also be written as

$\left(\boldsymbol{I}+\boldsymbol{A}_{\mathrm{I}}^{\prime}\right) \boldsymbol{g}_{\mathrm{I}}=\boldsymbol{G}_{\mathrm{s}}, \quad$ in $\mathrm{D}_{\infty}^{(d)}$,

where the equivalent interface response operator is

$\boldsymbol{A}_{\mathrm{I}}^{\prime}=\boldsymbol{A}_{\mathrm{s}}^{\prime}+\boldsymbol{G}_{\mathrm{s}} \boldsymbol{V}_{\mathrm{I}}, \quad$ in $\mathrm{D}_{\infty}^{(d)}$.

It is clear then that the general equation (2.82) has an alternative equivalent general form

$\left(\boldsymbol{I}+\boldsymbol{A}^{\prime}\right) \boldsymbol{g}=\boldsymbol{G}$,

with an equivalent to eq. (2.89) interface response operator

$\boldsymbol{A}^{\prime}=\boldsymbol{A}_{\mathrm{s} N}^{\prime}+\boldsymbol{G}\left(\boldsymbol{V}_{\mathrm{I}}+\mathbf{V}_{\mathrm{p}}\right)$,

and the same definitions for $\boldsymbol{G}, \boldsymbol{V}_{\mathrm{I}}$ and $\boldsymbol{V}_{\mathrm{p}}$ as before.

\section{A few general applications}

\subsection{Introduction}

In section 2 were obtained two general and equivalent equations

$$
\begin{array}{ll}
\boldsymbol{g}(\boldsymbol{I}+\boldsymbol{A})=\boldsymbol{G}, & \text { in } \mathrm{D}^{(d)}, \\
\left(\boldsymbol{I}+\boldsymbol{A}^{\prime}\right) \boldsymbol{g}=\boldsymbol{G}, & \text { in } \mathrm{D}^{(d)},
\end{array}
$$

relating the response function $\boldsymbol{g}$ of any composite system to a reference block diagonal response function $\boldsymbol{G}$. This $\boldsymbol{G}$ can be constructed for some of its blocks out of the bulk response function of the corresponding infinite subsystems and for some others out of the ideal surface response function of these subsystems.

These response functions $\boldsymbol{g}$ and $\boldsymbol{G}$ are related through the interface response operators

$$
\begin{array}{ll}
\boldsymbol{A}=\boldsymbol{A}_{\mathrm{s}}+\left(\boldsymbol{V}_{\mathrm{I}}+\boldsymbol{V}_{\mathrm{p}}\right) \boldsymbol{G}, & \text { in } \mathbf{D}^{(d)}, \\
\text { or } & \\
\boldsymbol{A}^{\prime}=\boldsymbol{A}_{\mathrm{s}}^{\prime}+\boldsymbol{G}\left(\boldsymbol{V}_{\mathrm{I}}+\boldsymbol{V}_{\mathrm{p}}\right), & \text { in } \mathbf{D}^{(d)} .
\end{array}
$$

$\boldsymbol{A}_{\mathrm{s}}$ and $\boldsymbol{A}_{\mathrm{s}}^{\prime}$ are the block diagonal ideal surface response operators. Each block $A_{s}(i i)$ is defined in the space $D_{i}^{(d)}$ of the subsystem as in section 2.4. Remember that the block $\boldsymbol{A}_{\mathrm{s}}(i i) \equiv 0$, when the corresponding block in $\boldsymbol{G}$ is the ideal free surface $\boldsymbol{g}_{\mathrm{s}}(i i)$ rather than the bulk $\boldsymbol{G}_{\mathrm{s}}(i i)$.

$\boldsymbol{V}_{\mathrm{I}}$ is the operator which couples all the independent subsystems and $\boldsymbol{V}_{\mathrm{p}}$ is the operator which describes a perturbation (due to defects for example) of the 
operator $\boldsymbol{h}$ of the composite system. These two operators have non-vanishing elements only in the space $M^{(d)}$ of all interfaces.

These equations shall be used now to derive a few general applications of this theory. In section 3.2, two equivalent expressions will be given for the elements of the response function $\boldsymbol{g}$. Section 3.3 will deal with the new states due to the interfaces. Section 3.4 is devoted to the density of states and section 3.5 to the thermodynamical functions of any composite and perturbed system. Section 3.6 is about interface reflection and transmission and section 3.7 about the response to an external stimulus. Section 3.8 is on symmetries of the systcm and superperiodicities due to the interfaces.

\subsection{The elements of the response function}

\subsubsection{General relations}

In many applications the elements $\boldsymbol{g}\left(\boldsymbol{x}, \boldsymbol{x}^{\prime}\right),\left(\boldsymbol{x}, \boldsymbol{x}^{\prime}\right) \in \mathrm{D}^{(d)}$ of the response function $\boldsymbol{g}$ are needed. It is possible to obtain them as a function of the elements of the basic response function $\boldsymbol{G}$ and of those of the interface response operator $\boldsymbol{A}$ or $\boldsymbol{A}^{\prime}$. Remember that

$$
\begin{array}{llll}
\boldsymbol{A}\left(\boldsymbol{x}, \boldsymbol{x}^{\prime}\right) \equiv 0, & \text { for } & \boldsymbol{x} \notin \mathrm{M}^{(d)}, \\
\boldsymbol{A}^{\prime}\left(\boldsymbol{x}, \boldsymbol{x}^{\prime}\right) \equiv 0, & \text { for } & \boldsymbol{x}^{\prime} \notin \mathrm{M}^{(d)} .
\end{array}
$$

It is therefore helpful to introduce the following, in general "rectangular matrices"

$\boldsymbol{A}(\mathrm{MD})$ and $\boldsymbol{A}^{\prime}(\mathrm{DM})$,

which give all non-vanishing elements of $\boldsymbol{A}$ for $\boldsymbol{x} \in \mathbf{M}^{(d)}$ and $\boldsymbol{x}^{\prime} \in \mathrm{D}^{(d)}$ and thosc of $\boldsymbol{A}^{\prime}$ for $\boldsymbol{x} \in \mathrm{D}^{(d)}$ and $\boldsymbol{x}^{\prime} \in \mathrm{M}^{(d)}$.

The same notation can also be used for the other operators and especially the response functions $\boldsymbol{g}$ and $\boldsymbol{G}$.

Then eqs. (3.1) and (3.2) can be rewritten as

$$
\begin{aligned}
& \boldsymbol{g}(\mathrm{DD})+\boldsymbol{g}(\mathrm{DM}) \boldsymbol{A}(\mathrm{MD})=\boldsymbol{G}(\mathrm{DD}), \\
& \boldsymbol{g}(\mathrm{DD})+\boldsymbol{A}^{\prime}(\mathrm{DM}) \boldsymbol{g}(\mathrm{MD})=\boldsymbol{G}(\mathrm{DD}) .
\end{aligned}
$$

Let us introduce

$\Delta(\mathrm{MM})=\boldsymbol{I}(\mathrm{MM})+\mathbf{A}(\mathrm{MM})$,

$\Delta^{\prime}(\mathrm{MM})=I(\mathrm{MM})+\mathbf{A}^{\prime}(\mathrm{MM})$.

Then with the help of eqs. (3.7) and (3.8), one also has

$$
\boldsymbol{g}(\mathrm{DM}) \Delta(\mathrm{MM})=\boldsymbol{G}(\mathrm{DM}) \text {, }
$$

$\Delta^{\prime}(\mathrm{MM}) \boldsymbol{g}(\mathrm{MD})=\mathbf{G}(\mathrm{MD})$.

$\Delta$ and $\Delta^{\prime}$ are regular matrices within the interface space $M^{(d)}$. Their inverses 
$\Delta^{-1}$ and $\Delta^{-1}$ can be calculated as inverse matrices and relations (3.11) and (3.12) provide

$\boldsymbol{g}(\mathrm{DM})=\boldsymbol{G}(\mathrm{DM}) \Delta^{-1}(\mathrm{MM})$,

$\boldsymbol{g}(\mathrm{MD})=\Delta^{\prime-1}(\mathrm{MM}) \boldsymbol{G}(\mathrm{MD})$.

Putting these results into eqs. (3.7) and (3.8) one obtains two general results for the elements of the response function $\mathrm{g}$

$$
\begin{aligned}
& \boldsymbol{g}(\mathrm{DD})=\boldsymbol{G}(\mathrm{DD})-\boldsymbol{G}(\mathrm{DM}) \Delta^{-1}(\mathrm{MM}) \boldsymbol{A}(\mathrm{MD}) \\
& \boldsymbol{g}(\mathrm{DD})=\boldsymbol{G}(\mathrm{DD})-\mathbf{A}^{\prime}(\mathrm{DM}) \Delta^{\prime-1}(\mathrm{MM}) \boldsymbol{G}(\mathrm{MD})
\end{aligned}
$$

\subsubsection{Summary and other relations between $\boldsymbol{g}$ and $\mathbf{G}$}

The elements of the response function $\boldsymbol{g}$ are given as a function of the elements of the reference response function $\boldsymbol{G}$ and of the interface response operators $\boldsymbol{A}$ and $\boldsymbol{A}^{\prime}$ by the general equations (3.15) and (3.16). The elements of $\boldsymbol{g}$ for which one point is in the interface domain $\mathrm{M}^{(d)}$ can even be more directly obtained out of eqs. (3.13) and (3.14).

Let us also remark that by mutual combination of eqs. (3.13)-(3.16) many other relations between the elements of $\boldsymbol{g}$ and $\boldsymbol{G}$ can be worked out. It is in particular possible to eliminate in some of these relations the explicit dependence on $\boldsymbol{A}$ and $\boldsymbol{A}^{\prime}$.

It is in particular straightforward to show with the help of eqs. (3.13) and (3.15) or (3.14) and (3.16), that

$$
\begin{aligned}
\boldsymbol{g}(\mathrm{DD})= & \boldsymbol{G}(\mathrm{DD})+\boldsymbol{g}(\mathrm{DM}) \boldsymbol{g}^{-1}(\mathrm{MM}) \boldsymbol{g}(\mathrm{MD}) \\
& -\boldsymbol{G}(\mathrm{DM}) \boldsymbol{G}^{-1}(\mathrm{MM}) \boldsymbol{G}(\mathrm{MD})
\end{aligned}
$$

In section 3.4 this relation will prove to be useful for the study of the variations of the total density of states.

However, for the calculation of the elements of $\boldsymbol{g}$, eqs. (3.13)-(3.16) seem to be the simplest ones as they give the elements of $\boldsymbol{g}$ as a function only of the known functions $\boldsymbol{G}$ and $\boldsymbol{A}$ (or $\boldsymbol{A}^{\prime}$ ).

\subsection{Interface states}

Many operators take the following form $\boldsymbol{H}_{0}=\boldsymbol{E} \boldsymbol{I}-\hat{\boldsymbol{H}}_{0}, \quad$ in $\mathrm{D}_{\infty}^{(d)}$.

The diagonalization of $\hat{\boldsymbol{H}}_{0}$ in discrete spaces provides the eigenvalues $E$ of this operator.

From the definition (2.1) of the resolvant operator $\boldsymbol{g}_{0}$ associated to $\boldsymbol{H}_{0}$, it is obvious that when $\boldsymbol{H}_{0}$ has the form (3.18) that $\boldsymbol{G}_{0}$ is also a function of $E$ and that the poles of $\boldsymbol{G}_{0}(E)$ are the eigenvalues of $\hat{\boldsymbol{H}}_{0}$. 
Similarly, the corresponding operator $\boldsymbol{h}$ of any real system with interfaces, can be written as

$\boldsymbol{h}=\boldsymbol{E} \boldsymbol{I}-\hat{\boldsymbol{h}}, \quad$ in $\mathrm{D}^{(d)}$,

in the space $\mathrm{D}^{(d)}$ of definition of the real system under study.

And the poles of the resolvant operator $\boldsymbol{g}(E)$ associated to $\boldsymbol{h}$ by

$\boldsymbol{h} \cdot \boldsymbol{g}=\boldsymbol{l}$, in $\mathrm{D}^{(d)}$,

will obviously be the eigenvalues of $\hat{\boldsymbol{h}}$.

Returning now to eqs. (3.13)-(3.16), one sees at once that the poles of $g$ are the poles of $\boldsymbol{G}$ plus those of $\Delta^{-1}$ or $\Delta^{\prime-1}$. So the eigenvalues of the operator $\hat{\boldsymbol{h}}$ of any real system are the eigenvalues of the bulk operators $\hat{H}_{0}(i i)$ if the corresponding subsystems are represented by the bulk $\boldsymbol{G}_{\mathrm{s}}(i i)$ in $\boldsymbol{G}$, and those of the ideal surface operators $\boldsymbol{h}_{\mathrm{s}}(i i)$ if the corresponding subsystems are represented by the surface $\boldsymbol{g}_{\mathrm{s}}(\mathrm{ii})$ in $\boldsymbol{G}$, plus new eigenvalues due to the poles of $\Delta^{-1}$ or $\Delta^{\prime-1}$. These new poles are then given by

$\operatorname{det}|\Delta(E)|=0, \quad$ in $\mathbf{M}^{(d)}$,

or

$\operatorname{det}\left|\Delta^{\prime}(E)\right|=0, \quad$ in $\mathrm{M}^{(d)}$.

Remember that $\Delta(E)$ and $\Delta^{\prime}(E)$ are defined within the interface space $\mathrm{M}^{(d)}$. Then these new poles of $\boldsymbol{g}$ are the new interface eigenvalues or eigenstates of the composite system.

\subsection{Density of states}

\subsubsection{Total densities of states versus response functions}

For the operator given by eq. (3.18) it is usual to define a total bulk density of states per unit volume by

$n_{0 i}(E)=\operatorname{Tr} \delta\left(\boldsymbol{H}_{0}(i, i)\right)$, in $\mathrm{D}_{\infty}^{(d)}$,

where $\operatorname{Tr}$ is the usual notation for the trace of a matrix and $\delta\left(\hat{\boldsymbol{H}}_{0}\right)$ is the usual Kronecker's $\delta$ function of $\hat{\boldsymbol{H}}_{0}$. The index $i$ reminds one that it is the bulk density of states of the $i$ subsystem.

Define

$\boldsymbol{G}_{0}^{ \pm}(E)=\lim _{\epsilon \rightarrow 0} \boldsymbol{G}_{0}(E \pm \mathrm{i} \epsilon), \quad$ in $\mathrm{D}_{\infty}^{(d)}$,

and use the symbolic relationship

$\lim _{\epsilon \rightarrow 0} \frac{I}{X+i \epsilon I}=\lim _{\epsilon \rightarrow 0} \frac{x}{x^{2}+I \epsilon^{2}}-i \pi \delta(\boldsymbol{x})$

to show that

$n_{0 i}(E)=-\pi^{-1} \operatorname{Im} \operatorname{Tr} \boldsymbol{G}_{0}^{+}(i i ; E), \quad$ in $\mathrm{D}_{\infty}^{(d)}$. 
In the same manner as in eq. (3.22), one defines the total density of states of any composite system per unit volume by

$n(E)=\operatorname{Tr} \delta(\hat{\boldsymbol{h}}), \quad$ in $\mathrm{D}^{(d)}$.

And as above, after having defined

$g^{+}(E)=\lim _{\epsilon \rightarrow 0} \boldsymbol{g}(E+\mathrm{i} \epsilon), \quad$ in $\mathrm{D}^{(d)}$,

one can write

$n(E)=-\pi^{-1} \operatorname{Im} \operatorname{Tr} g^{+}(E), \quad$ in $\mathrm{D}^{(d)}$.

\subsubsection{Local densities of states}

Considering eq. (3.28) one sees that the total density of states $n(E)$ is the sum of local densities of states $n(E, \boldsymbol{x})$ :

$n(E)=\sum_{x} n(E, x), \quad x \in \mathrm{D}^{(d)}$,

with

$n(E, x)=-\pi^{-1} \operatorname{Im} g^{+}(E \mid x, x), \quad x \in \mathrm{D}^{(d)}$.

In particular eqs. (3.30) and (3.13) or (3.14) enable one to easily calculate the local density of states at an interface point

$$
\begin{aligned}
& n(E, x)=-\frac{1}{\pi} \operatorname{Im} \sum_{x^{\prime}} G^{+}\left(x, x^{\prime}\right) \Delta^{-1}\left(x^{\prime}, x\right), \quad\left\{x, x^{\prime}\right\} \in \mathbb{M}^{(d)}, \\
& \text { or } \\
& n(E, x)=-\frac{1}{\pi} \operatorname{Im} \sum_{x^{\prime}} \Delta^{\prime}{ }^{1}\left(x, x^{\prime}\right) G\left(x^{\prime}, x\right), \quad\left\{x, x^{\prime}\right\} \in M^{(d)} .
\end{aligned}
$$

\subsubsection{Variation of the total density of states}

\subsubsection{General result}

Another useful entity is the variation of the total density of states between the reference system represented by $\boldsymbol{G}^{+}(E)$ and the real composite system represented by $\boldsymbol{g}^{+}(E)$.

Remember that the reference response function $\mathbf{G}^{+}$may be constructed for some of the subsystems $i$ out of a block of bulk response functions $\boldsymbol{G}_{\mathrm{s}}^{+}(i i)$ in $\mathrm{D}_{i}^{(d)}\left(1 \leqq i \leqq N^{\prime}\right)$ and for other subsystems $i^{\prime}$ out of a block of surface response functions $\boldsymbol{g}_{\mathrm{s}}^{+}\left(i^{\prime} i^{\prime}\right)$ in $\mathrm{D}_{i^{\prime}}^{(d)}\left(N^{\prime}<i^{\prime} \leqq N\right)$. What then is the physical meaning of

$n_{\mathrm{R}}(E)=-\pi^{-1} \operatorname{Im} \operatorname{Tr} \boldsymbol{G}^{+}(E)$, in $\mathrm{D}^{(d)}$, 
or

$n_{\mathrm{R}}(E)=-\frac{1}{\pi} \sum_{i=1}^{N^{\prime}} \operatorname{Im} \operatorname{Tr} \boldsymbol{G}_{\mathrm{s}}^{+}(i i)-\frac{1}{\pi} \sum_{i^{\prime}=N^{\prime}+1}^{N} \operatorname{Im} \operatorname{Tr} \boldsymbol{g}_{\mathrm{s}}^{+}\left(i^{\prime} i^{\prime}\right), \quad$ in $\mathrm{D}^{(d)}$.

The first term in eq. (3.34) is nothing else than the sum of the total bulk density of states per unit volume of all $i$ subsystems. This tends to exactness when the number of particles contained in the finite domain $D_{i}^{(d)}$ is large enough to be able to replace the Tr or summation over $\boldsymbol{x}$ by an integration over the volume of $\mathrm{D}_{i}^{(d)}$. This cannot be done for subsystems having a small number of particles, one adatom for example. For such subsystems, it is easier to obtain directly the surface response function $\boldsymbol{g}_{\mathrm{s}}\left(i^{\prime} i^{\prime}\right)$ which for the example given is nothing else than the response function of the isolated atom before adsorption. The second term in eq. (3.34) gives therefore the densities of states per unit volume of all free surface finite subsystems $i^{\prime}$. It is indeed more meaningful for subsystems with a small number of particles to use as reference this surface density of states rather than the bulk density of states of the corresponding infinite system.

Having clarified the meaning of the reference total density of states $n_{\mathrm{R}}(E)$ per unit volume of the composite system, one may now ask: How can the variation of the total density of states (eqs. (3.28) and (3.33))

$n(E)-n_{\mathrm{R}}(E)=-\pi^{-1} \operatorname{Im} \operatorname{Tr}\left|\boldsymbol{g}^{+}(E)-\boldsymbol{G}^{+}(E)\right|, \quad$ in $\mathrm{D}^{(d)}$,

between the composite system and the corresponding reference one be obtained?

With the help of eq. (3.17), this density of states variation can be written as $n(E)-n_{\mathrm{R}}(E)=-\pi^{-1}(t-T), \quad$ in $\mathrm{D}^{(d)}$,

with

$t=\operatorname{Im} \operatorname{Tr}\left|\boldsymbol{g}(\mathrm{DM}) \boldsymbol{g}^{-1}(\mathrm{MM}) \boldsymbol{g}(\mathrm{MD})\right|, \quad$ in $\mathrm{D}^{(d)}$,

and

$T=\operatorname{Im} \operatorname{Tr}\left|\boldsymbol{G}(\mathrm{DM}) \boldsymbol{G}^{-1}(\mathrm{MM}) \boldsymbol{G}(\mathrm{MD})\right|, \quad$ in $\mathrm{D}^{(d)}$.

In expressions (3.37) and (3.38) and in what follows, we omit to write explieitly the index + in $\boldsymbol{g}^{+}$and $\boldsymbol{G}^{+}$.

Note that $\boldsymbol{G}$ is formed out of independent blocks for each subsystem $D_{i}^{(d)}$. Therefore

$T=\sum_{i=1}^{N^{\prime}} T_{1}(i)+\sum_{i^{\prime}=N^{\prime}+1}^{N} T_{2}\left(i^{\prime}\right), \quad$ in $\mathrm{D}^{(d)}$,

with

$T_{1}(i)=\operatorname{Im} \operatorname{Tr}\left|\boldsymbol{G}_{\mathrm{s}}\left(\mathrm{D}_{i} \mathrm{M}_{i}\right) \boldsymbol{G}_{\mathrm{s}}^{-1}\left(\mathrm{M}_{i} \mathbf{M}_{i}\right) \boldsymbol{G}_{\mathrm{s}}\left(\mathrm{M}_{i} \mathrm{D}_{i}\right)\right|$ 
and

$T_{2}\left(i^{\prime}\right)=\operatorname{Im} \operatorname{Tr}\left|\mathbf{G}_{\mathrm{s}}\left(\mathrm{D}_{i^{\prime}} \mathrm{M}_{i^{\prime}}\right) \boldsymbol{g}_{\mathrm{s}}^{-1}\left(\mathrm{M}_{i^{\prime}} \mathrm{M}_{i^{\prime}}\right) \boldsymbol{G}_{\mathrm{s}}\left(\mathrm{M}_{i^{\prime}} \mathrm{D}_{i^{\prime}}\right)\right|, \quad$ in $\mathrm{D}^{(\mathrm{d})}$.

$T_{1}(i)$ is formed out of the $G_{s}(i i)$ which are truncated within $\mathrm{D}_{i}^{(d)}$ bulk response functions $\boldsymbol{G}_{0}(i i)$. To such truncated operators, the theorem of the cyclic invariance of the trace does not in general apply. Then $T_{1}(i)$ has to be calculated in the form of eq. (3.39b). $T_{2}\left(i^{\prime}\right)$ is formed out of the ideal surface response functions $\boldsymbol{g}_{\mathrm{s}}(i i)$ which are complete within $\mathrm{D}_{i}^{(d)}$. This term has the same form as $t$ in eq. (3.37), which is formed out of the complete response function $\boldsymbol{g}$ within $\mathrm{D}^{(d)}$. Therefore, analyse $t$ and the result can be easily transposed for $T_{2}\left(i^{\prime}\right)$.

Apply to the complete within $\mathrm{D}^{(d)}$ operator appearing in the right-hand side of eq. (3.37) the theorem of the cyclic invariance of the trace

$t=\operatorname{Im} \operatorname{Tr}\left|\boldsymbol{g}^{-1}(\mathrm{MM}) \boldsymbol{g}(\mathrm{MD}) \boldsymbol{g}(\mathrm{DM})\right|$.

Take the derivative versus $E$ of eq. (3.20), where $\boldsymbol{h}$ has the form of eq. (3.19), to obtain

$\boldsymbol{g}(\mathrm{MD}) \boldsymbol{g}(\mathrm{DM})=-\mathrm{d} \boldsymbol{g}(\mathrm{MM}) / \mathrm{d} E$.

Use the general property of any operator (or matrix) $B$

$\operatorname{Tr} \boldsymbol{B}^{-1} \mathrm{~d} B / \mathrm{d} E=\mathrm{d}(\ln \operatorname{det}|\boldsymbol{B}|) / \mathrm{d} E$,

and rewrite with the help of these last two properties, eq. (3.40) as

$t=-\operatorname{Im} \mathrm{d}(\ln \operatorname{det}|\boldsymbol{g}(\mathrm{MM})|) / \mathrm{d} E$,

or

$\mathrm{t}=-\mathrm{d}(\arg \operatorname{det}|\boldsymbol{g}(\mathrm{MM})|) / \mathrm{d} E$.

With the help of eq. (3.11) this is equivalent to

$t=\mathrm{d}[\arg \operatorname{det}|\Delta(\mathrm{MM})|-\arg \operatorname{det}|\mathbf{G}(\mathrm{MM})|] / \mathrm{d} E$,

and remembering that $\boldsymbol{G}$ is a block diagonal matrix

$t=\mathrm{d}\left[\arg \operatorname{det}|\Delta(\mathbf{M M})|-\sum_{i=1}^{N} \arg \operatorname{det}\left|\mathbf{G}\left(\mathbf{M}_{i} \mathbf{M}_{i}\right)\right|\right] / \mathrm{d} E$,

where

$\boldsymbol{G}\left(\mathrm{M}_{i} \mathrm{M}_{i}\right)=\left\{\begin{array}{lll}\boldsymbol{G}_{\mathrm{s}}\left(\mathrm{M}_{i} \mathrm{M}_{i}\right)=\boldsymbol{G}_{0}\left(\mathrm{M}_{i} \mathrm{M}_{i}\right) & \text { for } & 1 \leqq i \leqq N^{\prime}, \\ \boldsymbol{g}_{\mathrm{s}}\left(\mathrm{M}_{i} \mathrm{M}_{i}\right) & \text { for } & N^{\prime}<i \leqq N .\end{array}\right.$

Repeating the same demonstration as above for $T_{2}\left(i^{\prime}\right)$, eq. $(3.39 \mathrm{c})$, one also obtains

$T_{2}\left(i^{\prime}\right)=-\mathrm{d}\left(\arg \operatorname{det}\left|g_{\mathrm{s}}\left(\mathrm{M}_{i^{\prime}} \mathrm{M}_{i^{\prime}}\right)\right|\right) / \mathrm{d} E$. 
Finally going back to eq. (3.36) with the results (3.39), (3.46) and (3.48) provides

$n(E)-n_{\mathrm{R}}(E)-\frac{1}{\pi} \sum_{i=1}^{N^{\prime}}\left[T_{1}(i)+\frac{\mathrm{d}}{\mathrm{d} E} \arg \operatorname{det}\left|\boldsymbol{G}_{\mathrm{s}}\left(\mathbf{M}_{i} \mathbf{M}_{i}\right)\right|\right]+\frac{1}{\pi} \frac{\mathrm{d} \eta(E)}{\mathrm{d} E}$

where

$\eta(E)=-\arg \operatorname{det}|\Delta(\mathrm{MM})|$,

and $T_{1}(i)$ is given by eq. $(3.39 \mathrm{~b})$.

Note that when going from eq. (3.44) to eq. (3.45), it is possible to use eq. (3.14) rather than eq. (3.13).

Then, the general result (3.49) remains valid with

$\eta(E)=-\arg \operatorname{det}\left|\Delta^{\prime}(\mathrm{MM})\right|$.

\subsubsection{Particular results}

3.4.3.2.1. In the complementary to $D_{i}^{(d)}$ space. In order to seek for a simpler expression of $T_{1}(i)$, eq. $(3.39 \mathrm{~b})$, define $\mathrm{D}_{i \mathrm{c}}^{(d)}$ as the space complementary to $\mathrm{D}_{i}^{(d)}$ in $\mathrm{D}_{\infty}^{(d)}$ and $\mathrm{D}_{i \mathrm{M}}^{(d)}$ as the space $\mathrm{D}_{i}^{(d)}$ without $\mathrm{M}_{i}^{(d)}$. Then for the bulk response function $\boldsymbol{G}_{0}\left(\right.$ ii) in $\mathrm{D}_{\infty}^{(d)}$ one may write

$$
\begin{aligned}
& \operatorname{Im} \operatorname{Tr}\left|G_{0}\left(D_{i} M_{i}\right) G_{0}{ }^{1}\left(M_{i} M_{i}\right) G_{0}\left(M_{i} D_{i}\right)\right| \\
& =T_{1}(i)+\operatorname{Im} \operatorname{Tr}\left|G_{0}\left(D_{i c} M_{i}\right) G_{0}^{-1}\left(M_{i} M_{i}\right) G_{0}\left(M_{i} D_{i c}\right)\right| \\
& T_{1}(i)=\operatorname{Im} \operatorname{Tr} \mid G_{0}\left(M_{i} M_{i}\right) G_{0}^{-1}\left(M_{i} M_{i}\right) G_{0}\left(M_{i} M_{i}\right) \\
& \quad+G_{0}\left(D_{i M} M_{i}\right) G_{0}^{-1}\left(M_{i} M_{i}\right) G_{0}\left(M_{i} D_{i M}\right) \mid
\end{aligned}
$$

The left-hand side part of eq. (3.52) can also be transformed as $t$ from eq. (3.37) to eq. (3.44). Then eq. (3.52) becomes

$$
\begin{aligned}
T_{1}(i)= & -\mathrm{d}\left(\arg \operatorname{det} \boldsymbol{G}_{0}\left(\mathbf{M}_{i} \mathbf{M}_{i}\right)\right) / \mathrm{d} E \\
& -\operatorname{Im} \operatorname{Tr}\left|\boldsymbol{G}_{0}\left(\mathrm{D}_{i \mathrm{c}} \mathbf{M}_{i}\right) \mathbf{G}_{0}^{-1}\left(\mathbf{M}_{i} \mathbf{M}_{i}\right) \boldsymbol{G}_{0}\left(\mathbf{M}_{i} \mathrm{D}_{i \mathrm{c}}\right)\right|
\end{aligned}
$$

This expression of $T_{1}(i)$ may be easier to compute that those of eq. (3.39b), when in particular the extension in space of $\mathrm{D}_{i c}^{(d)}$ is smaller than those of its complementary $\mathrm{D}_{i}^{(d)}$.

Note also that eq. (3.53) can be obviously rewritten as

$T_{1}(i)=\operatorname{Im} \operatorname{Tr} \boldsymbol{G}_{0}\left(\mathrm{M}_{i} \mathrm{M}_{i}\right)+\operatorname{Im} \operatorname{Tr}\left|\boldsymbol{G}_{0}\left(\mathrm{D}_{i \mathrm{M}} \mathbf{M}_{i}\right) \boldsymbol{G}_{0}^{-1}\left(\mathrm{M}_{i} \mathbf{M}_{i}\right) \boldsymbol{G}_{0}\left(\mathbf{M}_{i} \mathrm{D}_{i \mathrm{M}}\right)\right|$ 
3.4.3.2.2. The case of specular symmetry through $M_{i}^{(d)}$. In the special case when the infinite system $i$ has specular symmetry through the interface $\mathbf{M}_{i}^{(d)}$, then the last terms in eqs. (3.54) and (3.55) are equal and then

$T_{1}(i)=\frac{1}{2} \operatorname{Im} \operatorname{Tr} \boldsymbol{G}_{0}\left(\mathbf{M}_{i} \mathbf{M}_{i}\right)-\frac{1}{2} \mathrm{~d}\left(\arg \operatorname{det}\left|\boldsymbol{G}_{0}\left(\mathbf{M}_{i} \mathbf{M}_{i}\right)\right|\right) / \mathrm{d} E$.

The general result of eq. (3.49) takes for those subsystems $i$ with specular symmetry through the interface $\mathrm{M}_{i}^{(d)}$ in $\mathrm{D}_{\infty}^{(d)}$ a much simpler form.

3.4.3.2.3. When one uses only surface response functions in $\mathbf{G}$. There exist cases where it is simpler to construct the whole reference response function $\mathbf{G}$ out of surface response functions $\boldsymbol{g}_{\mathrm{s}}(i i)$. Then the general result (3.49) takes the simple form

$n(E)-n_{\mathrm{R}}(E)=\pi^{-1} \mathrm{~d} \eta(E) / \mathrm{d} E$.

This is also the case when one studies as a perturbation a defect in an infinite system, then one uses the bulk response function $\boldsymbol{G}_{0}$ of this system and $\boldsymbol{A}$ reduces to just $\boldsymbol{V}_{\mathrm{p}} \boldsymbol{G}_{0}$ and $\boldsymbol{A}^{\prime}$ to $\boldsymbol{G}_{0} \boldsymbol{V}_{\mathrm{p}}$.

In these cases, one has here in $D_{\infty}^{(d)}$ the well known in $D_{\infty}^{(3)}$ phase shift $\eta(E)$ method.

Note also that if a new interface state given by eq. (3.21a) appeared at a value of $E=E_{l}$ at which

$\arg \operatorname{det}|\Delta(\mathrm{MM})|=n \pi, \quad n=0,1, \ldots$,

then eq. (3.57) implies that $\eta(E)$ must show a discontinuous jump of $+\pi$ at $E=E_{l}$. Similarly if a discrete state $E=E_{\mathrm{d}}$ (due to one adatom for example) disappeared when going from the reference system to the real one, then $\eta(E)$ must show a discontinuous jump of $-\pi$ at $E=E_{\mathrm{d}}$.

\subsubsection{Conservation of the number of states}

When the number of independent degrees of freedom is not changed when going from the reference system to the real composite one, then the total number of states is conserved. This implies that

$\int_{-\infty}^{+\infty}\left|n(E)-n_{\mathrm{R}}(E)\right| \mathrm{d} E=0$.

This is the most usual case. One may however wish to compare two systems which differ in their degrees of freedom (addition or removal of particles for example). This can also be studied within the frame of this theory, but one must count in the second member of eq. (3.58) the number $N_{\mathrm{g}}$ of degrees of freedom gained or lost.

In particular, when the variation of the density of states takes the simple form of eq. (3.57), then the above considerations imply

$\frac{1}{\pi} \int_{-\infty}^{+\infty} \mathrm{d} E \frac{\mathrm{d} \eta(E)}{\mathrm{d} E}=N_{\mathrm{g}}$ 
or

$\pi^{-1}|\eta(+\infty)-\eta(-\infty)|=N_{\mathrm{g}}$.

\subsection{Variations of additive functions}

Define an additive function $F$ of the composite system by

$F=\int_{-\infty}^{+\infty} \mathrm{d} E f(E) n(E)$

and $F_{\mathrm{R}}$ of the reference system by

$F_{\mathrm{R}}=\int_{-\infty}^{+\infty} \mathrm{d} E f(E) n_{\mathrm{R}}(E)$.

In these expressions $f(E)$ may be any function of $E$ and of other parameters, like the temperature and the pressure $p . F$ and $F_{\mathrm{R}}$ can be for example usual thermodynamical functions, like the free energy, the specific heat, the entropy, ....

Define the variation of the additive functions between the real and the reference system by

$F-F_{\mathrm{R}}=\int_{-\infty}^{+\infty} \mathrm{d} E f(E)\left|n(E)-n_{\mathrm{R}}(E)\right|$.

So the general result (3.49) for the variation of the density of states enables the variation of any additive function to be calculated. These variations may be important physical entities like for example the surface and interface energies, specific heats, entropies, ....

In the special cases when the general result (3.49) reduces to the phase-shift one of eq. (3.57), the variation of $F$ becomes

$F-F_{\mathrm{R}}=\int_{-\infty}^{+\infty} \mathrm{d} E f(E) \frac{\mathrm{d} \eta(E)}{\mathrm{d} E}$.

Integrating by part, one obtains

$F-F_{\mathrm{R}}=\left.\frac{1}{\pi} f(E) \eta(E)\right|_{-\infty} ^{+\infty}-\frac{1}{\pi} \int_{-\infty}^{+\infty} \mathrm{d} E \frac{\mathrm{d} f(E)}{\mathrm{d} E} \eta(E)$.

When there is no change of the number of degrees of freedom between the reference and real systems $\left(N_{\mathrm{g}}=0\right)$, then with the help of eq. (3.60)

$F \quad F_{\mathrm{R}}=\frac{1}{\pi} \int_{-\infty}^{+\infty} \mathrm{d} E \frac{\mathrm{d} f(E)}{\mathrm{d}} \bar{E} \cdot \eta(E)$.

\subsection{Interface reflection and transmission}

Define the eigenvectors $|\psi\rangle$ and $|\Psi\rangle$ of the operators $\hat{h}$ and $\hat{\boldsymbol{H}}$ in 
respectively the composite and the reference systems. These eigenvectors are given by

$$
\begin{array}{ll}
(\hat{\boldsymbol{h}}-E \boldsymbol{I})|\Psi\rangle=0, & \text { in } \mathrm{D}^{(d)}, \\
(\hat{\boldsymbol{H}}-E \boldsymbol{I})|\Psi\rangle=0, & \text { in } \mathrm{D}^{(d)} .
\end{array}
$$

$\hat{\boldsymbol{H}}$ is the block diagonal operator of the reference system.

Compare now these two equations with the ones we solved before

$$
\begin{array}{ll}
(\hat{\boldsymbol{h}}-E \boldsymbol{I}) \boldsymbol{g}=\boldsymbol{I}, & \text { in } \mathrm{D}^{(d)}, \\
(\hat{\boldsymbol{H}}-\boldsymbol{E}) \boldsymbol{G}=\boldsymbol{I}, & \text { in } \mathrm{D}^{(d)} .
\end{array}
$$

The general demonstration of section 2, which led us to the results (3.15) and (3.16) from eqs. (3.66) and (3.67) can be repeated in the same manner when starting from eqs. (3.64) and (3.65). This enables us to write the following relations between the eigenvectors of the composite and the reference systems

$$
\begin{aligned}
& |\psi\rangle=|\Psi\rangle-\mathbf{A}^{\prime} \Delta^{-1}|\Psi\rangle, \\
& |\psi\rangle=|\Psi\rangle-\mathbf{G}^{-1} \mathbf{A} \boldsymbol{G}^{-1}|\Psi\rangle .
\end{aligned}
$$

In the same manner as in eqs. (3.15) and (3.16), $\mathbf{G}$ is formed out of blocks $i$ of either the bulk or the surface response functions within $\mathrm{D}_{i}^{(d)},|\Psi\rangle$ is also formed out of blocks $i$ of either the bulk or the surface eigenvectors within $\mathrm{D}_{i}^{(d)}$, respectively $\left|\Psi_{\mathrm{s}}(i)\right\rangle$ or $\left|\psi_{\mathrm{s}}(i)\right\rangle$ given by

$$
\left(\hat{\boldsymbol{H}}_{0}(i i)-E \boldsymbol{I}\right)\left|\Psi_{\mathrm{s}}(i)\right\rangle=0 \text {, }
$$

or

$$
\left(\hat{\boldsymbol{h}}_{\mathrm{s}}(i i)-E \boldsymbol{I}\right)\left|\psi_{\mathrm{s}}(i)\right\rangle=0 \text {. }
$$

Of course, in eqs. (3.68) and (3.69), the operators acting on $|\Psi\rangle$ have to be calculated in the same base as the eigenvectors $|\Psi\rangle$ and $|\psi\rangle$.

These relations between the eigenstates $|\Psi\rangle$ of the reference system built up of independent blocks and those $|\psi\rangle$ of the real composite system provide two equivalent manners to study the interface reflection and transmission.

For example when a wave represented by the bulk state $\left|\Psi_{s}(i)\right\rangle$ is launched on the interface $j$ separating the subsystem $i$ from another subsystem $i^{\prime}$, the corresponding reflection amplitude $f_{\mathrm{R}}$ from the eigenstate $\left|\Psi_{\mathrm{s}}(i)\right\rangle$ into another eigenstate $\left|\Psi_{s}^{\prime}(i)\right\rangle$ by the interface $j$ is

$$
\left\langle\Psi_{\mathrm{s}}^{\prime}(i j)\left|f_{\mathrm{R}}\right| \Psi_{\mathbf{s}}(i j)\right\rangle=-\left\langle\Psi_{\mathrm{s}}^{\prime}(i j)\left|\boldsymbol{A}^{\prime} \Delta^{\prime-1}\right| \Psi_{\mathrm{s}}(i j)\right\rangle, \quad \text { in } \mathbf{M}_{i j}^{(d)},
$$

or the equivalent expression one gets from eq. (3.69). In eq. (3.72) $|\Psi(i j)\rangle$ represents the value of the eigenstate $\left|\Psi_{s}(i)\right\rangle$ within $\mathbf{M}_{i j}^{(d)}$. When the wave $\left|\Psi_{s}(i)\right\rangle$ is for example a plane wave launched on a plane interface studied with eigenvalues taking into account the invariance of translation parallel to 
the interface, then in eq. (3.72) enters only one value for the $\left|\Psi_{\mathrm{s}}(i j)\right\rangle$. But for interfaces of general shape, the reflections by different points of the interface are not in general equivalent. From eqs. (3.68) and (3.69) one can study the reflection amplitude by one interface point or by composition the reflection amplitude by several or all points of the interface $j$ or even by all the interfaces bounding the $i$ subsystem.

In the same way, the transmission amplitude $f_{\mathrm{T}}$ from an eigenstate $\left|\Psi_{\mathrm{s}}(i)\right\rangle$ into an eigenstate $\left|\Psi_{s}^{\prime}\left(i^{\prime}\right)\right\rangle$ of any other subsystem $i^{\prime}$ through a common interface $j$ is

$$
\begin{aligned}
& \left\langle\Psi_{\mathrm{s}}^{\prime}\left(i^{\prime} j\right)\left|f_{\mathrm{T}}\right| \Psi_{\mathrm{s}}(i j)\right\rangle=-\left\langle\Psi_{\mathrm{s}}^{\prime}\left(i^{\prime} j\right)\left|\mathbf{A}^{\prime} \Delta^{\prime-1}\right| \Psi_{\mathrm{s}}(i j)\right\rangle, \\
& \quad \text { in }\left\{\mathbf{M}_{i j}^{(d)}, \mathbf{M}_{i^{\prime} j}^{(d)}\right\},
\end{aligned}
$$

or the equivalent expression one gets from eq. (3.69).

The same consideration as above for the reflection can be done here for the transmission. In particular eqs. (3.68) and (3.69) enables one to obtain the transmission from one subsystem $\mathrm{D}_{i}^{(d)}$ into any other (not necessarily adjacent to $\mathrm{D}_{i}^{(d)}$ ) subsystem $\mathrm{D}_{i^{\prime}}^{(d)}$.

As a summary define the equivalent scattering opcrators

$\boldsymbol{S}^{\prime}=-\boldsymbol{A}^{\prime} \boldsymbol{\Delta}^{\prime-1}$

$\mathbf{S}=-\boldsymbol{G} \Delta^{-1} \mathbf{A} \boldsymbol{G}^{-1}$,

which enables one to study any reflection or transmission from some eigenstates of the reference system into the same or other eigenstates of this reference system.

\subsection{Response to an external stimulus}

When the composite system under study is submitted to an external stimulus $|F(x)\rangle$ such that eq. (3.65) becomes

$\boldsymbol{h}|\Psi\rangle+|F\rangle=0$.

Then from eq. (3.66) the response to this external stimulus is

$$
|\Psi\rangle=-\boldsymbol{g}|F\rangle \text {. }
$$

This relation shows that the knowledge of the response operator $\boldsymbol{g}$ enables us to obtain the response $|\Psi\rangle$ of the system to any external stimulus $|F\rangle$.

\subsection{Symmetry and super-periodicity}

\subsubsection{Symmetries}

Before applying the above results to any specific system, it is very helpful to take advantage of all its symmetries like, for example, time invariance, time 
reversal, translation invariance along one or several space directions, reflection, rotation symmetries, all point group symmetries, .... In other words, it is very useful to use group theory to reduce as much as possible the dimensions of the operators $\boldsymbol{H}$ and $\boldsymbol{h}$, and hence $\boldsymbol{G}$ and $\boldsymbol{g}$ of respectively the reference and composite systems.

As the group theory methods to reduce the dimensions of operators and matrices are well known, it is not necessary to dwell on these methods here.

On the other hand, the supersymmetries due to periodical interfaces are less common and will be developed further in what follows.

\subsubsection{Interface super-periodicities}

Some composite systems are built out of periodical arrangements of two or more different subsystems. Consider such an infinite supersystem in $\mathrm{D}_{\infty}^{(d)}$. First the super unit cell of this system has to be precised. From this super-cell the whole infinite super system can be constructed by elementary translations.

Let $\boldsymbol{x}_{0}$ be any point within the super unit cell $\mathrm{D}_{\mathrm{u}}^{(d)}$ and $\boldsymbol{R}$ the general translation vector which enables the whole supersystem to be constructed

$\boldsymbol{R}=\sum_{l=1}^{d^{\prime}} n_{l} \boldsymbol{R}_{l}$,

where $\boldsymbol{R}_{l}$ is the unit translation vector in the direction $\boldsymbol{x}_{l}, n_{l}$ are positive or negative integers and $d^{\prime}\left(1 \leqq d^{\prime} \leqq d\right)$ gives the order of the super-periodicity.

Due to this super-translation symmetry, it is possible to define the following Fourier transforms

$$
\begin{aligned}
& \boldsymbol{g}\left(\boldsymbol{q} ; \boldsymbol{x}_{0}, \boldsymbol{x}_{0}^{\prime}\right)=\sum_{\boldsymbol{R}} \boldsymbol{g}\left(\boldsymbol{x}_{0}, \boldsymbol{x}_{0}^{\prime}+\boldsymbol{R}\right) \mathrm{e}^{\mathrm{i} \boldsymbol{q} \cdot \boldsymbol{R}}, \\
& \boldsymbol{G}\left(\boldsymbol{q} ; \boldsymbol{x}_{0}, \boldsymbol{x}_{0}^{\prime}\right)=\sum_{\boldsymbol{R}} \boldsymbol{G}\left(\boldsymbol{x}_{0}, \boldsymbol{x}_{0}^{\prime}+\boldsymbol{R}\right) \mathrm{e}^{\mathrm{i} \boldsymbol{q} \cdot \boldsymbol{R}}, \\
& \boldsymbol{A}\left(\boldsymbol{q} ; \boldsymbol{x}_{0}, \boldsymbol{x}_{0}^{\prime}\right)=\sum_{\boldsymbol{R}} \boldsymbol{A}\left(\boldsymbol{x}_{0}, \boldsymbol{x}_{0}^{\prime}+\boldsymbol{R}\right) \mathrm{e}^{\mathrm{i} \boldsymbol{q} \cdot \boldsymbol{R}}, \\
& \boldsymbol{A}^{\prime}\left(\boldsymbol{q} ; \boldsymbol{x}_{0}, \boldsymbol{x}_{0}^{\prime}\right)=\sum_{\boldsymbol{R}} \boldsymbol{A}^{\prime}\left(\boldsymbol{x}_{0}, \boldsymbol{x}_{0}^{\prime}+\boldsymbol{R}\right) \mathrm{e}^{\mathrm{i} \boldsymbol{\mathrm { q }} \cdot \boldsymbol{R}},
\end{aligned}
$$

where

$\left\{x_{0}, x_{0}^{\prime}\right\} \in \mathrm{D}_{\mathrm{u}}^{(d)}$,

$\mathrm{D}_{\mathrm{u}}^{(d)}$ being the super unit cell. Note also that from the above Fourier transforms of $\boldsymbol{A}$ and $\boldsymbol{A}^{\prime}$, one defines in the same manner $\Delta\left(\boldsymbol{q} ; \boldsymbol{x}_{0}, \boldsymbol{x}_{0}^{\prime}\right)$ and $\Delta^{\prime}\left(q ; x_{0}, x_{0}^{\prime}\right)$ within the space $\mathrm{M}_{\mathrm{u}}^{(d)}$ of those interfaces which are contained in the unit cell $\mathrm{D}_{\mathrm{u}}^{(d)}$.

The propagation vector $q$ is contained within the first Brillouin zone associated with the super unit cell. 
Finally the general equations (3.1) and (3.2) can be rewritten with the help of eqs. (3.79)-(3.82) as

$$
\begin{aligned}
& \boldsymbol{g}(\boldsymbol{q}) \cdot|\boldsymbol{I}+\boldsymbol{A}(\boldsymbol{q})|=\boldsymbol{G}(\boldsymbol{q}), \\
& \left|\boldsymbol{I}+\boldsymbol{A}^{\prime}(\boldsymbol{q})\right| \cdot \boldsymbol{g}(\boldsymbol{q})=\boldsymbol{G}(\boldsymbol{q}), \quad \text { in } \mathrm{D}_{\mathrm{u}}^{(d)} .
\end{aligned}
$$

All the other equations demonstrated in this section 3 can also be used for these Fourier transformed functions.

So for a supersystem, one can obtain from the Fourier transformed equations (3.15) or (3.16) the elements of $\boldsymbol{g}\left(\boldsymbol{q} ; \boldsymbol{x}_{0}, \boldsymbol{x}_{0}^{\prime}\right)$ in $\mathrm{D}_{\mathrm{u}}^{(d)}$. In the same manner, the Fourier transformed equations (3.21), will provide the eigenstates of the supersystem as a function of the propagation vector $q$. Similarly one may obtain the function of $q$ densities of states. One has to sum over $q$ if one wants the total densities of states and the variation of additive functions. One may also study other properties like interface reflection and transmission or response to external stimulus with this $\boldsymbol{g}\left(\boldsymbol{q} ; \boldsymbol{x}_{0}, \boldsymbol{x}_{0}^{\prime}\right)$ response function.

However if one wants to address problems associated for example with some effects due to defects or to free surfaces of supersystems, one needs to know the response function $\boldsymbol{g}$ in the whole $\mathrm{D}_{\infty}^{(d)}$ space. This function can be obtained by the inverse Fourier transformation of eq. (3.79)

$\boldsymbol{g}\left(\boldsymbol{x}_{0}, \boldsymbol{x}_{0}^{\prime}\right)=\sum_{\boldsymbol{q}} \boldsymbol{g}\left(\boldsymbol{q} ; \boldsymbol{x}_{0}, \boldsymbol{x}_{0}^{\prime}+\boldsymbol{R}\right) \mathrm{e}^{-\mathrm{i} \boldsymbol{q} \cdot \boldsymbol{R}}$.

However this Fourier transformation may not be the easiest way to obtain $g$ in the whole $\mathrm{D}_{\infty}^{(d)}$ space. Other techniques well known for the calculation of the response functions in infinite lattices may prove to be more efficient. For example the transfer matrix method may be easily adapted to the supersystem. In order to illustrate this, consider supersystems of dimension $d^{\prime}=1$. In this case $q$ is the propagation vector $k_{\perp}$ perpendicular to the interfaces and the real space translation vector

$\boldsymbol{R}=n \boldsymbol{R}_{\perp}$

is also perpendicular to the interfaces.

Suppose one has also translational invariance in directions parallel to the interfaces and one Fourier analyses also all operators appearing in eqs. (3.79)-(3.86) according to this invariance. All these operators become then a function of the propagation vector $k_{\|}$to the interfaces.

One can always write the operator $\boldsymbol{h}$ of such a supersystem in a general tridiagonal form by defining subblock diagonal parts in $\boldsymbol{h}$ interacting with just its first nearest neighbour subblocks.

Let $X_{03}$ and $X_{03}^{\prime}$ be the positions within the super unit cell of one of these blocks. In general $X_{03}$ represents a certain range of values of $X_{03}$. Then $\boldsymbol{A}, \boldsymbol{A}^{\prime}$, $\boldsymbol{G}$ and $\boldsymbol{g}$ can also be written within this $X_{3}$ representation as subblocks labelled by $X_{03}$ and $X_{03}^{\prime}$. 
Define then a transfer matrix $\boldsymbol{R}\left(\boldsymbol{k}_{\|}\right)$between two identical interfaces. Using its definition in eq. (3.1) provides us with matrix equations for $\boldsymbol{R}$. These equations can be solved inside $\mathbf{M}_{\mathrm{u}}^{(d)}$ and the transfer matrix is then obtained. Once these results are reported in the original matrix equation, one obtains the matrix elements of $\boldsymbol{g}$ between any two points in $\mathrm{D}_{\infty}^{(d)}$.

A similar procedure can also be used together with eq. (3.2).

More generally an infinite periodic supersystem is an infinite bulk system with a large unit cell. Then all the usual methods used for the study of bulk systems with small unit cells can be utilized for the study of such supersystems.

\section{Prospectives}

A new unified and simple theory of interface responses is proposed in this paper for any composite system in a $d$-dimensional discrete space.

It will be used in forthcoming papers to rederive in a simpler and unified manner many known analytical response functions and to obtain new ones as well. Many physical properties obtained with the help of these functions will be also given. This shall be done within lattice dynamics, electronic and magnetic theories of solids, for surfaces, interfaces, adsorbates, periodic interfaces (superlattices), membranes, defects, ....

\section{Short literature survey on crystal interface response functions}

The literature on response functions in discrete materials limited by a free surface is very large. Let us cite here only a few early papers [ 19$]$, review papers and books [10-13]. These functions provide also a suitable way for the study of adsorption $[5,7,11,12,13]$.

The surface response functions were used for the investigation of crystalline interfaces between two semi-infinite media, also first on simple phonon [14], electron [15] and magnon [16] models and then for more realistic ones; see for example the reviews [17-19].

A few papers appeared also on response functions in crystalline superlattices made out of a periodic repetition of two different slabs [20-23].

A recent theory of incomplete crystals, surfaces, defects, interfaces and layered structures is also in press [24].

\section{Acknowledgements}

During the preparation of this paper the author has benefited from very stimulating and helpful discussions with F. Garcia-Moliner and V. Velasco. 
He thanks them also for their hospitality and for communication of their unpublished crystal theories of defects, quantum well $\mathrm{ABC}$ and superlattices AB. He is deeply indebted to the Centre National de la Recherche Scientifique for their continuous support and for providing the necessary help during the author's stay in Madrid.

\section{References}

[1] L.N. Rosenzweig, Uch. Zap. Hark. Gos. Univ. Tr. Fiz. Mat. Otdel 2 (1950) 19.

[2] J. Koutecky, Phys. Rev. 108 (1957) 13.

[3] A.A. Maradudin and J. Melngailis, Phys. Rev. 133 (1964) A1188.

[4] R.A. Brown, Phys. Rev. 156 (1967) 889.

[5] L. Dobrzynski, Ann. Phys. (Paris) 4 (1969) 637.

[6] S.G. Davison and J.D. Levine, Solid State Phys. 25 (1970) 1.

[7] G. Allan, Ann. Phys. (Paris) 5 (1970) 169.

[8] S.W. Müsser and K.H. Rieder, Phys. Rev. B2 (1970) 3034.

[9] D. Kalstein and P. Soven, Surface Sci. 26 (1971) 85.

[10] A.A. Maradudin, E.W. Montroll, G.H. Weiss and I.P. Ipatova, Theory of Lattice Dynamics in the Harmonic Approximation (Academic Press, New York, 1963 and 1971).

[11] P. Lenglart, L. Dobrzynski and G. Leman, Ann. Phys. 7 (1972) 407.

[12] A.A. Maradudin, R.F. Wallis and L. Dobrzynski, in: Surface Phonons and Polaritons, Vol. 3 of the Handbook of Surfaces and Interfaces, Ed. L. Dobrzynski (Garland, New York, 1980).

[13] G. Allan, in: Handbook of Surfaces and Interfaces, Vol. 2, Ed. L. Dobrzynski (Garland, New York, 1978) p. 299.

[14] P. Masri and L. Dobrzynski, Surface Sci. 34 (1973) 119.

[15] E. Foo and H. Wang, Phys. Rev. B10 (1974) 4819.

[16] B. Djafari-Rouhani and L. Dobrzynski, J. Phys. (Paris) 36 (1975) 835.

[17] B. Djafari-Rouhani, L. Dobrzynski and P. Masri, Ann. Phys. (Paris) 6 (1981) 259.

[18] J. Pollman, Advan. Solid State Phys. 20 (1980) 117.

[19] J. Pollman and A. Mazur, Thin Solid Films 104 (1983) 257.

[20] L. Dobrzynski, B. Djafari-Rouhani and O. Hardouin Duparc, J. Electron Spectrosc. Related Phenomena 30 (1983) 119.

[21] B. Djafari-Rouhani, L. Dobrzynski and P. Masri, Phys. Rev. B31 (1985) 7739.

[22] P. Masri, L. Dobrzynski, B. Djafari-Rouhani and J.O.A. Idiodi, Surface Sci. 166 (1986) 301.

[23] L. Dobrzynski, B. Djafari-Rouhani and H. Puszkarski, Phys. Rev. B33 (1986) 3251.

[24] F. Garcia-Moliner and V. Velasco, Progr. Surface Sci., in press. 